\title{
PROVABLY PHYSICAL-CONSTRAINT-PRESERVING DISCONTINUOUS GALERKIN METHODS FOR MULTIDIMENSIONAL RELATIVISTIC MHD EQUATIONS
}

\author{
KAILIANG WU* AND CHI-WANG SHU†
}

February 7, 2020

\begin{abstract}
We propose and analyze a class of robust, uniformly high-order accurate discontinuous Galerkin (DG) schemes for multidimensional relativistic magnetohydrodynamics (RMHD) on general meshes. A distinct feature of the schemes is their physical-constraint-preserving (PCP) property, i.e., they are proven to preserve the subluminal constraint on the fluid velocity and the positivity of density, pressure, and specific internal energy. Developing PCP high-order schemes for RMHD is highly desirable but remains a challenging task, especially in the multidimensional cases, due to the inherent strong nonlinearity in the constraints and the effect of the magnetic divergence-free condition. Inspired by some crucial observations at the PDE level, we construct the provably PCP schemes by using the locally divergence-free DG schemes of the recently proposed symmetrizable RMHD equations as the base schemes, a limiting technique to enforce the PCP property of the DG solutions, and the strong-stability-preserving methods for time discretization. We rigorously prove the PCP property by using a novel "quasi-linearization" approach to handle the highly nonlinear physical constraints, technical splitting to offset the influence of divergence error, and sophisticated estimates to analyze the beneficial effect of the additional source term in the symmetrizable RMHD system. Several two-dimensional numerical examples are provided to confirm the PCP property and to demonstrate the accuracy, effectiveness and robustness of the proposed PCP schemes.
\end{abstract}

Key words. relativistic magnetohydrodynamics, discontinuous Galerkin method, physical-constraint-preserving, high-order accuracy, locally divergence-free, hyperbolic conservation laws

AMS subject classifications. 65M60, 65M12, 35L65, 76W05

1. Introduction. This paper is concerned with developing robust high-order accurate numerical methods for the special relativistic magnetohydrodynamics (RMHD) equations, which are used to describe the dynamics of electrically-conducting fluids at nearly the speed of light in the presence of magnetic field. RMHD play an important role in many fields, such as astrophysics and high energy physics, and have been used to investigate a number of astrophysical scenarios from stellar to galactic scales, e.g., gamma-ray bursts, formation of black holes, astrophysical jets, blast waves of supernova explosions, gravitational collapse and accretion, etc.

The special RMHD equations are often formulated as a nonlinear system of hyperbolic conservation laws

$$
\mathbf{U}_{t}+\nabla \cdot \mathbf{F}(\mathbf{U})=\mathbf{0}
$$

where $\nabla \cdot=\sum_{i=1}^{d} \frac{\partial}{\partial x_{i}}$ is the divergence operator with $d \in\{1,2,3\}$ denoting the spatial dimensionality. Here we employ the geometrized unit system so that the speed of light $c=1$. In (1), the conservative vector $\mathbf{U}=(D, \mathbf{m}, \mathbf{B}, E)^{\top}$, and the flux $\mathbf{F}=\left(\mathbf{F}_{1}, \ldots, \mathbf{F}_{d}\right)$ is defined by

$$
\mathbf{F}_{i}(\mathbf{U})=\left(D v_{i}, v_{i} \mathbf{m}-B_{i}\left(W^{-2} \mathbf{B}+(\mathbf{v} \cdot \mathbf{B}) \mathbf{v}\right)+p_{t o t} \mathbf{e}_{i}, v_{i} \mathbf{B}-B_{i} \mathbf{v}, m_{i}\right)^{\top},
$$

with the mass density $D=\rho W$, the momentum vector $\mathbf{m}=\left(\rho H W^{2}+|\mathbf{B}|^{2}\right) \mathbf{v}-(\mathbf{v} \cdot \mathbf{B}) \mathbf{B}$, the magnetic field $\mathbf{B}=\left(B_{1}, B_{2}, B_{3}\right)$, the energy $E=\rho H W^{2}-p_{\text {tot }}+|\mathbf{B}|^{2}$, and the vector $\mathbf{e}_{i}$ denoting the $i$-th row of the unit matrix of size 3. Additionally, $\rho$ is the rest-mass density, $\mathbf{v}=\left(v_{1}, v_{2}, v_{3}\right)$ denotes the velocity field of the fluid, $W=1 / \sqrt{1-|\mathbf{v}|^{2}}$ is the Lorentz factor, $p_{t o t}$ is the total pressure consisting of the thermal pressure $p$ and the magnetic pressure $p_{m}:=\frac{1}{2}\left(W^{-2}|\mathbf{B}|^{2}+(\mathbf{v} \cdot \mathbf{B})^{2}\right), H=1+e+\frac{p}{\rho}$ represents the specific enthalpy, and $e$ is the specific internal energy. The equation of state (EOS) is needed to close the system (1). A general EOS can be expressed as

$$
H=H(p, \rho) .
$$

\footnotetext{
*Department of Mathematics, The Ohio State University, Columbus, OH 43210, USA (wu.3423@osu.edu).

${ }^{\dagger}$ Division of Applied Mathematics, Brown University, Providence, RI 02912, USA (Chi-Wang_Shu@brown.edu.). Research is supported in part by NSF grant DMS-1719410.
} 
A simple example is the ideal EOS

$$
H=1+\frac{\Gamma p}{(\Gamma-1) \rho}
$$

where $\Gamma \in(1,2]$ is a constant and denotes the adiabatic index, for which the restriction $\Gamma \leq 2$ is required by the compressibility assumptions and the relativistic causality. Given an EOS, the conservative vector $\mathbf{U}$ and the flux $\mathbf{F}$ can be explicitly expressed by the primitive variables $\{\rho, p, \mathbf{v}, \mathbf{B}\}$. However, unlike the non-relativistic case, there are no explicit formulas for either the flux $\mathbf{F}$ or the primitive variables $\{\rho, p, \mathbf{v}\}$ in terms of $\mathbf{U}$, due to the relativistic effect, especially the appearance of the Lorentz factor.

The magnetic field should also satisfy an additional divergence-free condition

$$
\nabla \cdot \mathbf{B}:=\sum_{i=1}^{d} \frac{\partial B_{i}}{\partial x_{i}}=0,
$$

which is a reflection of the principle that there are no magnetic monopoles. Although the satisfaction of (4) is not explicitly included in the system (1), the exact solution of (1) always preserves zero divergence for $\mathbf{B}$ in future time if the initial divergence is zero. Besides the standard difficulty in solving the nonlinear hyperbolic systems, an additional numerical challenge for the RMHD system (1) comes from the divergence-free condition (4), which is also involved in the ideal non-relativistic MHD system. It is widely realized that the condition (4) is important for robust computations, since large divergence error in the numerical magnetic field can lead to numerical instabilities or nonphysical structures in the computed solutions, cf. $[9,2,30,17]$. In the one-dimensional case $(d=1), B_{1}$ is constant so that the condition (4) can be easily enforced in numerical computations. However, in the multidimensional cases $(d \geq 2)$, numerical preservation of (4) is highly nontrivial, and various techniques have been proposed to reduce the divergence error or enforce the divergence-free condition in the discrete sense; see e.g., [9, 23, 30, 6, 29, 17, 18, 43, 10] and the references therein.

In physics, the density, thermal pressure and internal energy are positive, and the fluid velocity must be slower than the speed of light in the vacuum $c=1$. Mathematically, an equivalent description is that the conservative vector $\mathbf{U}$ must stay in the set of physically admissible states defined by

$$
\mathcal{G}:=\left\{\mathbf{U}=(D, \mathbf{m}, \mathbf{B}, E)^{\top}: \rho(\mathbf{U})>0, p(\mathbf{U})>0, e(\mathbf{U})>0,|\mathbf{v}(\mathbf{U})|<1\right\},
$$

where the functions $\rho(\mathbf{U}), p(\mathbf{U}), e(\mathbf{U})$ and $\mathbf{v}(\mathbf{U})$ are highly nonlinear and cannot be explicitly formulated in terms of $\mathbf{U}$, due to the relativistic effect. In numerical computations, preserving the numerical solutions in $\mathcal{G}$ is highly desirable and crucial for the robustness of the numerical schemes. This is because once any physical constraints in (5) are violated in the numerical simulations, the discrete problem becomes ill-posed due to the loss of hyperbolicity, causing the breakdown of the simulation codes. In the past several decades, various numerical schemes have been developed for the RMHD, e.g., [15, 7, 21, 31, 13, 44, 1, 49]. However, none of them were proven to preserve all these constraints, even though they have been used to simulate some RMHD flows successfully. In fact, most of the existing RMHD schemes do not always preserve these constraints, and thus may suffer from a large risk of failure when simulating RMHD problems with large Lorentz factor, low density or pressure, or strong discontinuity. It is therefore highly significant and desirable to develop physical-constraint-preserving $(\mathrm{PCP})$ numerical schemes whose solutions always stay in the set $\mathcal{G}$.

During the past decade, significant progress has been made for constructing bound-preserving high-order accurate schemes for hyperbolic systems, mainly built on two types of limiters. One is a simple scaling limiter for the reconstructed or evolved solution polynomials in finite volume or discontinuous Galerkin (DG) methods; see, e.g., [46, 47, 45, 35, 37, 50]. Another one is a fluxcorrection limiter, see, e.g., [42, 14, 19, 3]. For more developments, we refer interested readers to the survey [27] and references therein. With these limiting approaches, several PCP methods were developed for the special relativistic hydrodynamics (RHD) without the magnetic field, including high-order accurate PCP finite difference schemes [38], PCP DG schemes [24], PCP central DG schemes [40], and PCP Lagrangian finite volume schemes [20]. Extension of the PCP methods from 
special to general RHD is highly nontrivial. An earlier effort [26] was made in this direction but only enforced the positivity of density. Recently, frameworks of designing provably PCP high-order finite difference, finite volume and DG methods were established in [33] for the general RHD. All of the aforementioned PCP methods were restricted to RHD without the magnetic field.

Seeking PCP schemes for the RMHD is highly challenging, largely due to the intrinsic complexity of the RMHD equations and strong nonlinearity contained in the physical constraints in (5). As mentioned above, there are no explicit expressions of the highly nonlinear functions $\rho(\mathbf{U}), p(\mathbf{U})$, $e(\mathbf{U})$ and $\mathbf{v}(\mathbf{U})$ for the RMHD. Taking the ideal EOS case (3) as example, in order to obtain the values of $\{\rho, p, e, \mathbf{v}\}$ from a given vector $\mathbf{U}=(D, \mathbf{m}, \mathbf{B}, E)^{\top}$, one has to solve a nonlinear algebraic equation [21]:

$$
\theta-\frac{\Gamma-1}{\Gamma}\left(\frac{\theta}{\Upsilon_{\mathbf{U}}^{2}(\theta)}-\frac{D}{\Upsilon_{\mathbf{U}}(\theta)}\right)+|\mathbf{B}|^{2}-\frac{1}{2}\left(\frac{|\mathbf{B}|^{2}}{\Upsilon_{\mathbf{U}}^{2}(\theta)}+\frac{(\mathbf{m} \cdot \mathbf{B})^{2}}{\theta^{2}}\right)-E=0,
$$

for the unknown $\theta \in \mathbb{R}^{+}$, where the function $\Upsilon_{\mathbf{U}}(\theta)$ is defined by

$$
\Upsilon_{\mathbf{U}}(\theta)=\left(\frac{\theta^{2}\left(\theta+|\mathbf{B}|^{2}\right)^{2}-\left[\theta^{2}|\mathbf{m}|^{2}+\left(2 \theta+|\mathbf{B}|^{2}\right)(\mathbf{m} \cdot \mathbf{B})^{2}\right]}{\theta^{2}\left(\theta+|\mathbf{B}|^{2}\right)^{2}}\right)^{-1 / 2} .
$$

Assume that an admissible solution of the equation (6) exists for the given state $\mathbf{U}$, and denote it by $\hat{\theta}=\hat{\theta}(\mathbf{U})$, then the primitive variables in (5) can be computed by

$$
\begin{array}{lr}
\mathbf{v}(\mathbf{U})=\left(\mathbf{m}+\hat{\theta}^{-1}(\mathbf{m} \cdot \mathbf{B}) \mathbf{B}\right) /\left(\hat{\theta}+|\mathbf{B}|^{2}\right), & \rho(\mathbf{U})=\frac{D}{\Upsilon_{\mathbf{U}}(\hat{\theta})}, \\
p(\mathbf{U})=\frac{\Gamma-1}{\Gamma \Upsilon_{\mathbf{U}}^{2}(\hat{\theta})}\left(\hat{\theta}-D \Upsilon_{\mathbf{U}}(\hat{\theta})\right), & e(\mathbf{U})=\frac{p(\mathbf{U})}{(\Gamma-1) \rho(\mathbf{U})} .
\end{array}
$$

As clearly shown in the above procedure, checking the admissibility of a given state $\mathbf{U}$ is already a very difficult task. On the other hand, in most of the numerical RMHD schemes, the conservative quantities are themselves evolved according to their own conservation laws, which are seemingly unrelated to and numerically do not necessarily guarantee the desired bounds of the computed primitive variables $\{\rho, p, e, \mathbf{v}\}$. In theory, it is indeed a challenge to make an a priori judgment on whether a scheme is always PCP under all circumstances or not. Therefore, the study of PCP schemes for the RMHD has remained blank until the recent work in [39], where several important mathematical properties of the set $\mathcal{G}$ were first derived and PCP finite volume and DG methods were developed for the conservative RMHD equations (1) in one space dimension. Moreover, for the multidimensional conservative RMHD equations, the theoretical analysis in [39] revealed that the PCP property of standard finite volume and DG methods is closely connected with a discrete divergence-free condition on the numerical magnetic field. This finding was further extended on general meshes in [41] and is consistent with the ideal non-relativistic MHD case [34]. It was also shown in [39, 41] that if the discrete divergence-free condition is slightly violated, even the first-order multidimensional Lax-Friedrichs scheme for (1) is not PCP in general. Unfortunately, the required discrete divergence-free condition relies on certain combination of the information on adjacent cells, so that it could not be naturally enforced by any existing divergence-free techniques that also work in conjunction with the standard local scaling PCP limiter [39]. Therefore, the design of multidimensional PCP schemes for the RMHD has challenges essentially different from the one-dimensional case. As a result, provably PCP high-order schemes have not yet been obtained for the conservative RMHD system (1) in the multidimensional cases.

The focus of this paper is to develop a class of provably PCP high-order DG schemes for the multidimensional RMHD with a general EOS on general meshes. Towards achieving this goal, we will make the following efforts in this paper:

1. First, we investigate the PCP property of the exact solutions of the conservative RMHD system (1) at the PDE level. We observe that, if the divergence-free condition (4) is (slightly) violated, the 
exact smooth solution of (1) may fail to be PCP, i.e., $\mathcal{G}$ is not an invariant region for the exact solution of (1). Therefore, before seeking provably PCP numerical schemes, our first task is to reformulate the RMHD equations so as to accommodate the PCP property at the PDE level. We consider a symmetrizable formulation of the RMHD equations, which we recently proposed in [36], by building the divergence-free condition (4) into the equations through adding a source term. We show that the exact smooth solutions of the symmetrizable RMHD system always retain the PCP property even if the magnetic field is not divergence-free.

2. Based on the symmetrizable formulation, we establish a framework of constructing provably PCP high-order DG schemes for the multidimensional RMHD with a general EOS on general meshes. The key is to properly discretize the symmetrizable RMHD equations so as to eliminate the effect of divergence error on the PCP property of the resulting DG schemes. We adopt the locally divergencefree DG elements, which enforce zero divergence within each cell, and a suitable discretization of the symmetrization source term, which brings some discrete divergence terms into our schemes and exactly offsets the influence of divergence error on the PCP property.

3. A significant innovation in this paper is that we discover and rigorously prove the PCP property of the proposed DG schemes, without requiring any discrete divergence-free condition. There are two main technical challenges in the proof. One is how to explicitly and analytically verify the admissibility of any given conservative state $\mathbf{U}$, without solving the nonlinear equation (6). This difficulty has been addressed in [39] based on two equivalent forms of the admissible state set $\mathcal{G}$. The other is how to take the advantages of the locally divergence-free property and our suitable discretization of the source term in the symmetrizable RMHD formulation, to eliminate the effect of divergence error on the PCP property. Due to the locally divergence-free property and the source term, the limiting values of the numerical solution at the interfaces of each cell are intrinsically coupled, making some standard analysis techniques ([47]) inapplicable. We will overcome this difficulty by using a novel "quasi-linearization" approach to handle the highly nonlinear constraints in (5), technical splitting to offset the influence of divergence error, and sophisticated estimates to analyze the beneficial effect of the symmetrization source term.

4. We implement the proposed PCP DG schemes on two-dimensional Cartesian meshes and demonstrate their accuracy, effectiveness and robustness for several numerical examples. We will show that our PCP schemes, without any artificial treatments, are able to successfully simulate several challenging problems, including a strongly magnetized bast problem with extremely low plasma-beta $\left(2.5 \times 10^{-10}\right)$ and highly supersonic RMHD jets, which are rarely considered in the literature.

The present study is also motivated by our recent work [35,37] on the positivity-preserving DG schemes for the ideal non-relativistic MHD. Compared to the non-relativistic case, the present study is much more challenging, due to the highly nonlinear coupling of the RMHD equations and the complicated mapping from the conservative to primitive variables. Additional technical challenges also arise from the suitable discretization of the symmetrization source term and especially some novel estimate techniques required to analyze its beneficial effect on the PCP property.

2. Auxiliary observations on the PCP property at the PDE level. This section introduces our observations on the PCP property of the exact smooth solutions of the conservative formulation (1) and a symmetrizable formulation of the RMHD equations, respectively, with the ideal EOS (3). The findings will provide some insights that guide us to successfully construct the PCP schemes for the RMHD.

We observe that negative pressure may appear in the exact smooth solution of the conservative RMHD system (1) if $\nabla \cdot \mathbf{B} \neq 0$. An evidence, rather than rigorous proof, for this claim may be given by considering the following initial condition

$$
\begin{aligned}
& \rho(\mathbf{x}, 0)=1, \quad p(\mathbf{x}, 0)=1-\exp \left(-|\mathbf{x}|^{2}\right), \\
& \mathbf{v}(\mathbf{x}, 0)=(0.01,0.01,0.01), \quad \mathbf{B}(\mathbf{x}, 0)=\left(1+\delta B_{1}, 1+\delta B_{2}, 1+\delta B_{d}\right),
\end{aligned}
$$

where $\mathbf{x}=\left(x_{1}, \ldots, x_{d}\right)$, and $\delta B_{i}=\epsilon \arctan x_{i}, 1 \leq i \leq 3$, are small perturbations with $0<\epsilon \ll 1$. Since the initial solution (8) is bounded and infinitely differentiable, it is reasonable to assume: there exists a small time interval $\left[0, t_{*}\right)$ such that the exact solution of the system (1) with (8) 
exists and is smooth for $t \in\left[0, t_{*}\right)$. Since $|\mathbf{v}(\mathbf{0}, 0)|-1=-0.97<0$ and $\rho(\mathbf{0}, 0)=1>0$, by the sign-preserving property for continuous functions, there exists a neighborhood $\Omega$ of $\mathbf{0}$ in $\mathbb{R}^{d}$ and $t_{0} \in\left(0,, t_{*}\right)$ such that $|\mathbf{v}(\mathbf{x}, t)|-1<0$ and $\rho(\mathbf{x}, t)>0$ for all $(\mathbf{x}, t) \in \Omega \times\left[0, t_{0}\right)$. Let us then study the initial time derivative of $p \rho^{-\Gamma}$ at $(\mathbf{x}, t)=(\mathbf{0}, 0)$. For smooth solutions, we derive from (1) that $\frac{\partial}{\partial t}\left(p \rho^{-\Gamma}\right)+\mathbf{v} \cdot \nabla\left(p \rho^{-\Gamma}\right)+(\Gamma-1) \rho^{-\Gamma}(\mathbf{v} \cdot \mathbf{B}) \nabla \cdot \mathbf{B}=0$. At $(\mathbf{x}, t)=(\mathbf{0}, 0)$, we have $\nabla\left(p \rho^{-\Gamma}\right)=\mathbf{0}$ and $\nabla \cdot \mathbf{B}=d \epsilon>0$, which yield $\frac{\partial\left(p \rho^{-\Gamma}\right)}{\partial t}(\mathbf{0}, 0)=-0.03 d(\Gamma-1) \epsilon<0$. Note that $p \rho^{-\Gamma}(\mathbf{0}, 0)=0$. Thus there exists $t_{1} \in\left[0, t_{0}\right)$ such that $p \rho^{-\Gamma}(\mathbf{0}, t)<0, \forall t \in\left(0, t_{1}\right)$. Because $\rho(\mathbf{x}, t)>0$ for all $(\mathbf{x}, t) \in \Omega \times\left[0, t_{0}\right)$, we have $p(\mathbf{0}, t)<0, \forall t \in\left(0, t_{1}\right)$.

The above analysis infers that the exact smooth solution of the conservative RMHD system (1) may fail to be PCP if the divergence-free condition (4) is violated. This observation, along with the results in [39] at the numerical level, demonstrate the unity of continuous and discrete objects, and clearly reveal the intrinsic connection between the PCP property and divergence-free condition. In most of the numerical RMHD schemes including the standard DG methods, the divergence error in magnetic field is generally unavoidable, although there exist a few numerical techniques to enforce exactly or globally divergence-free property (e.g., $[18,43,10])$. On the other hand, the standard PCP limiting technique (cf. [47, 39]) with local scaling can destroy the globally divergence-free property. It is therefore difficult to find a numerical technique which can enforce the globally divergence-free property and meet the PCP requirement at the same time. In order to address the above issue, we propose to consider a symmetrizable formulation of the RMHD equations [36]

$$
\mathbf{U}_{t}+\nabla \cdot \mathbf{F}(\mathbf{U})=-\mathbf{S}(\mathbf{U}) \nabla \cdot \mathbf{B}
$$

where

$$
\mathbf{S}(\mathbf{U}):=\left(0,\left(1-|\mathbf{v}|^{2}\right) \mathbf{B}+(\mathbf{v} \cdot \mathbf{B}) \mathbf{v}, \mathbf{v}, \mathbf{v} \cdot \mathbf{B}\right)^{\top} .
$$

The system (9) is analogous to the Godunov-Powell system [11, 22] for the ideal non-relativistic MHD. The right-hand side term of (9) is proportional to $\nabla \cdot \mathbf{B}$. This implies, at the continuous level, the two formulations (9) and (1) are equivalent under the condition (4). However, the "source term" $\mathbf{S}(\mathbf{U}) \nabla \cdot \mathbf{B}$ in (9) modifies the character of the equations, making the system (9) symmetrizable, admit a convex thermodynamic entropy pair, and play a key role in designing entropy stable schemes [36]. These good properties do not hold for the conservative RMHD system (1).

Interestingly, we find that the exact smooth solutions of the symmetrizable RMHD system (9) always retain the desired PCP property at the PDE level, even if the divergence-free condition (4) is not satisfied. Consider the initial-value problem of the system (9), for $\mathbf{x} \in \mathbb{R}^{d}$ and $t>0$, with initial data

$$
(\rho, \mathbf{v}, p, \mathbf{B})(\mathbf{x}, 0)=\left(\rho_{0}, \mathbf{v}_{0}, p_{0}, \mathbf{B}_{0}\right)(\mathbf{x}),
$$

where the magnetic field is not necessarily divergence-free. Using the method of characteristics one can show the following result, whose proof is given in Appendix A.

Proposition 2.1. Assume the initial data (11) are in $C^{1}\left(\mathbb{R}^{d}\right)$ with $\rho_{0}(\mathbf{x})>0, p_{0}(\mathbf{x})>0$, and $\left|\mathbf{v}_{0}(\mathbf{x})\right|<1, \forall \mathbf{x} \in \mathbb{R}^{d}$. If the initial-value problem of (9) with (11) has a $C^{1}$ solution $(\rho, \mathbf{v}, p, \mathbf{B})(\mathbf{x}, t)$ for $\mathbf{x} \in \mathbb{R}^{d}$ and $0 \leq t \leq T$, then the solution satisfies

$$
\rho(\mathbf{x}, t)>0, p(\mathbf{x}, t)>0, e(\mathbf{x}, t)>0,|\mathbf{v}(\mathbf{x}, t)|<1, \quad \forall \mathbf{x} \in \mathbb{R}^{d}, \forall t \in[0, T] .
$$

In addition, if assuming the solution is $C^{2}$, then it holds

$$
\min _{\mathbf{x} \in \mathbb{R}^{d}} \frac{\nabla \cdot \mathbf{B}}{\rho W}(\mathbf{x}, 0) \leq \frac{\nabla \cdot \mathbf{B}}{\rho W}(\mathbf{x}, t) \leq \max _{\mathbf{x} \in \mathbb{R}^{d}} \frac{\nabla \cdot \mathbf{B}}{\rho W}(\mathbf{x}, 0), \quad \forall t \in[0, T] .
$$

For smooth solutions of the modified RMHD system (9), the estimate (12) implies that the "relative" divergence $\left\|\rho^{-1} W^{-1} \nabla \cdot \mathbf{B}(\cdot, t)\right\|_{L^{\infty}}$ does not grow with $t$.

Analogous to the Powell source term for the ideal non-relativistic MHD system [23, 35, 37], the source term in the symmetrizable RMHD system (9) is non-conservative, but is necessary to accommodate the PCP property at the PDE level when the divergence-free condition (4) is not 
exactly satisfied. Therefore, in order to achieve the PCP property at the discrete level, our schemes in this paper will be constructed using the symmetrizable formulation (9), which renders additional technical challenges in discretizing the source term properly to ensure its compatibility with the PCP property. As mentioned in $[35,37]$ on the non-relativistic MHD, there is a conflict between the PCP property which requires the non-conservative source term, and the conservation property which is lost due to the source term. The loss of conservation property leaves the possibility that it may lead to incorrect resolutions for some discontinuous problems, which will be investigated carefully in a separate study.

3. Numerical analysis techniques. In this section, we will introduce several important properties of $\mathcal{G}$ and derive some technical estimates, which will be useful in the PCP analysis of the proposed numerical schemes.

3.1. Properties of admissible states. Throughout the rest of this paper, we consider a general causal EOS (2) satisfying

$$
\begin{cases}\text { The function } H(p, \rho) \text { in }(2) \text { is differentiable in } \mathbb{R}^{+} \times \mathbb{R}^{+}, \\ H(p, \rho) \geq \sqrt{1+p^{2} / \rho^{2}}+p / \rho, & \forall p, \rho>0, \\ H(p, \rho)\left(\frac{1}{\rho}-\frac{\partial H(p, \rho)}{\partial p}\right)<\frac{\partial H(p, \rho)}{\partial \rho}<0, & \forall p, \rho>0,\end{cases}
$$

where the second condition is revealed by the relativistic kinetic theory [40], and the third condition can be derived from the relativistic causality and the assumption that the fluid's coefficient of thermal expansion is positive [40], which is valid for most compressible fluids, e.g., the gases. The conditions in (13) are valid for the ideal EOS (3) and some other commonly used EOSs; see [40].

In order to overcome the challenges arising from the lack of explicit formulas of the functions in (5), the following two equivalent forms of $\mathcal{G}$ were rigorously derived in [39] for the ideal EOS (3) and in [41] for a general EOS (2) satisfying (13).

Lemma 3.1 (First equivalent form). The admissible state set $\mathcal{G}$ is equivalent to the set

$$
\mathcal{G}_{1}:=\left\{\mathbf{U}=(D, \mathbf{m}, \mathbf{B}, E)^{\top}: D>0, q(\mathbf{U})>0, \Psi(\mathbf{U})>0\right\},
$$

where $q(\mathbf{U}):=E-\sqrt{D^{2}+|\mathbf{m}|^{2}}$ and

$$
\Psi(\mathbf{U}):=\left(\Phi(\mathbf{U})-2\left(|\mathbf{B}|^{2}-E\right)\right) \sqrt{\Phi(\mathbf{U})+|\mathbf{B}|^{2}-E}-\sqrt{\frac{27}{2}\left(D^{2}|\mathbf{B}|^{2}+(\mathbf{m} \cdot \mathbf{B})^{2}\right)},
$$

with $\Phi(\mathbf{U}):=\sqrt{\left(|\mathbf{B}|^{2}-E\right)^{2}+3\left(E^{2}-D^{2}-|\mathbf{m}|^{2}\right)}$.

Lemma 3.2 (Second equivalent form). The admissible state set $\mathcal{G}$ or $\mathcal{G}_{1}$ is equivalent to the set

$$
\mathcal{G}_{2}:=\left\{\mathbf{U}=(D, \mathbf{m}, \mathbf{B}, E)^{\top}: D>0, \mathbf{U} \cdot \boldsymbol{\xi}^{*}+p_{m}^{*}>0, \forall \mathbf{B}^{*} \in \mathbb{R}^{3}, \forall \mathbf{v}^{*} \in \mathbb{B}_{1}(\mathbf{0})\right\},
$$

where $\mathbb{B}_{1}(\mathbf{0}):=\left\{\mathbf{x} \in \mathbb{R}^{3}:|\mathbf{x}|<1\right\}$ denotes the open unit ball centered at $\mathbf{0}$ in $\mathbb{R}^{3}$, and

$$
\begin{aligned}
& \boldsymbol{\xi}^{*}=\left(-\sqrt{1-\left|\mathbf{v}^{*}\right|^{2}},-\mathbf{v}^{*},-\left(1-\left|\mathbf{v}^{*}\right|^{2}\right) \mathbf{B}^{*}-\left(\mathbf{v}^{*} \cdot \mathbf{B}^{*}\right) \mathbf{v}^{*}, 1\right)^{\top}, \\
& p_{m}^{*}=\frac{\left(1-\left|\mathbf{v}^{*}\right|^{2}\right)\left|\mathbf{B}^{*}\right|^{2}+\left(\mathbf{v}^{*} \cdot \mathbf{B}^{*}\right)^{2}}{2} .
\end{aligned}
$$

Remark 3.3. Note that all the constraints in the above two equivalent forms are explicit with respect to $\mathbf{U}$. This is a very helpful feature. The first equivalent form $\mathcal{G}_{1}$ is particularly useful for checking the admissibility of a given state $\mathbf{U}$ and constructing the limiter for developing PCP high-order accurate RMHD schemes. Moreover, the two constraints in the second equivalent form 
$\mathcal{G}_{2}$, are both linear with respect to $\mathbf{U}$, although two (additional) auxiliary variables $\mathbf{B}^{*}$ and $\mathbf{v}^{*}$ are introduced. Such linearity makes $\mathcal{G}_{2}$ quite suitable for analytically verifying the PCP property of RMHD schemes. It will provide a novel quasi-linearization approach to handle nonlinear physical constraints and play an important role in our PCP analysis.

It is also proven in [39] that the admissible state set is a convex set.

LEMma 3.4. The admissible state set $\mathcal{G}_{1}$ is convex.

3.2. Technical estimates. In order to handle the effect of the source term in the symmetrizable RMHD system (9) on the PCP property of numerical schemes, we derive the following inequality (17), whose discovery is highly nontrivial.

Lemma 3.5. For any $\mathbf{U} \in \mathcal{G}$, any $\mathbf{B}^{*} \in \mathbb{R}^{3}$ and any $\mathbf{v}^{*} \in \mathbb{B}_{1}(\mathbf{0})$, it holds

$$
\left|\mathbf{S}(\mathbf{U}) \cdot \boldsymbol{\xi}^{*}+\mathbf{v}^{*} \cdot \mathbf{B}^{*}\right| \leq \frac{1}{\sqrt{\rho H}}\left(\mathbf{U} \cdot \boldsymbol{\xi}^{*}+p_{m}^{*}\right),
$$

where $\boldsymbol{\xi}^{*}$ and $p_{m}^{*}$ are defined in (15) and (16), respectively.

Proof. We observe that

$$
\mathbf{S}(\mathbf{U}) \cdot \boldsymbol{\xi}^{*}+\mathbf{v}^{*} \cdot \mathbf{B}^{*}=\left(\mathbf{v}-\mathbf{v}^{*}\right) \cdot\left(\left(1-|\mathbf{v}|^{2}\right) \mathbf{B}+(\mathbf{v} \cdot \mathbf{B}) \mathbf{v}-\left(1-\left|\mathbf{v}^{*}\right|^{2}\right) \mathbf{B}^{*}-\left(\mathbf{v}^{*} \cdot \mathbf{B}^{*}\right) \mathbf{v}^{*}\right) .
$$

Let $\Pi_{1}:=\mathbf{U} \cdot \boldsymbol{\xi}^{*}+p_{m}^{*}$ and

$$
\Pi_{2}:=\sqrt{\rho H}\left(\mathbf{v}-\mathbf{v}^{*}\right) \cdot\left(\left(1-|\mathbf{v}|^{2}\right) \mathbf{B}+(\mathbf{v} \cdot \mathbf{B}) \mathbf{v}-\left(1-\left|\mathbf{v}^{*}\right|^{2}\right) \mathbf{B}^{*}-\left(\mathbf{v}^{*} \cdot \mathbf{B}^{*}\right) \mathbf{v}^{*}\right) .
$$

Then, we need to prove

$$
\Pi_{1} \geq\left|\Pi_{2}\right| .
$$

We reformulate $\Pi_{1}$ and split it into two parts as follows:

$$
\begin{aligned}
\Pi_{1}= & \rho H W^{2}\left(1-\mathbf{v} \cdot \mathbf{v}^{*}\right)-p-\rho W \sqrt{1-\left|\mathbf{v}^{*}\right|^{2}} \\
& +\left(|\mathbf{B}|^{2} \mathbf{v}-(\mathbf{v} \cdot \mathbf{B}) \mathbf{B}\right) \cdot\left(-\mathbf{v}^{*}\right)+\left(\left(1-\left|\mathbf{v}^{*}\right|^{2}\right) \mathbf{B}^{*}+\left(\mathbf{v}^{*} \cdot \mathbf{B}^{*}\right) \mathbf{v}^{*}\right) \cdot(-\mathbf{B}) \\
& +\frac{\left(1+|\mathbf{v}|^{2}\right)|\mathbf{B}|^{2}-(\mathbf{v} \cdot \mathbf{B})^{2}}{2}+\frac{\left(1-\left|\mathbf{v}^{*}\right|^{2}\right)\left|\mathbf{B}^{*}\right|^{2}+\left(\mathbf{v}^{*} \cdot \mathbf{B}^{*}\right)^{2}}{2} \\
= & {\left[\rho H W^{2}\left(1-\mathbf{v} \cdot \mathbf{v}^{*}\right)-p-\rho W \sqrt{1-\left|\mathbf{v}^{*}\right|^{2}}\right] } \\
& +\left[\frac{\left(1-\left|\mathbf{v}^{*}\right|^{2}\right)\left|\mathbf{B}-\mathbf{B}^{*}\right|^{2}}{2}+\frac{\left|\mathbf{v}-\mathbf{v}^{*}\right|^{2}|\mathbf{B}|^{2}}{2}-\frac{\left(\left(\mathbf{v}-\mathbf{v}^{*}\right) \cdot \mathbf{B}\right)^{2}}{2}+\frac{\left(\mathbf{v}^{*} \cdot\left(\mathbf{B}-\mathbf{B}^{*}\right)\right)^{2}}{2}\right] \\
= & : \Pi_{1}^{(1)}+\Pi_{1}^{(2)} .
\end{aligned}
$$

According to the second condition in (13), the first part $\Pi_{1}^{(1)}$ satisfies

$$
\begin{aligned}
\frac{\Pi_{1}^{(1)}}{\rho H} & =W^{2}\left(1-\mathbf{v} \cdot \mathbf{v}^{*}\right)-\frac{p}{\rho H}-\frac{1}{H} W \sqrt{1-\left|\mathbf{v}^{*}\right|^{2}} \geq W^{2}\left(1-\mathbf{v} \cdot \mathbf{v}^{*}\right)-\frac{\frac{H^{2}-1}{2 H} \rho}{\rho H}-\frac{1}{H} W \sqrt{1-\left|\mathbf{v}^{*}\right|^{2}} \\
& =W^{2}\left(1-\mathbf{v} \cdot \mathbf{v}^{*}\right)-\frac{1}{2}+\frac{1}{2}\left(\frac{1}{H}-W \sqrt{1-\left|\mathbf{v}^{*}\right|^{2}}\right)^{2}-\frac{W^{2}\left(1-\left|\mathbf{v}^{*}\right|^{2}\right)}{2} \\
& \geq W^{2}\left(1-\mathbf{v} \cdot \mathbf{v}^{*}\right)-\frac{1}{2}-\frac{W^{2}\left(1-\left|\mathbf{v}^{*}\right|^{2}\right)}{2}=\frac{1}{2} W^{2}\left|\mathbf{v}-\mathbf{v}^{*}\right|^{2} .
\end{aligned}
$$

It follows that

$$
\Pi_{1} \geq \frac{1}{2} \rho H W^{2}\left|\mathbf{v}-\mathbf{v}^{*}\right|^{2}+\Pi_{1}^{(2)}:=\Pi_{3} .
$$


Therefore, in order to prove (17) or (18), it suffices to show

$$
\Pi_{3} \geq\left|\Pi_{2}\right| .
$$

Let us introduce the vector $\mathbf{Z}=\left(\mathbf{B}^{*}, \mathbf{B}, \sqrt{\rho H}\right)^{\top} \in \mathbb{R}^{7}$. We observe that both $\Pi_{3}$ and $\Pi_{2}$ can be formulated into quadratic forms in the variables $\mathbf{Z}$. This highly nontrivial observation is a key of our proof. Specifically, we discover that

$$
\Pi_{3}=\frac{1}{2} \mathbf{Z}^{\top} \mathbf{A}_{3} \mathbf{Z}, \quad \Pi_{2}=\frac{1}{2} \mathbf{Z}^{\top} \mathbf{A}_{2} \mathbf{Z}
$$

with

$$
\mathbf{A}_{3}=\left(\begin{array}{ccc}
\mathbf{G} & -\mathbf{G} & \mathbf{0}^{\top} \\
-\mathbf{G} & \mathbf{H} & \mathbf{0}^{\top} \\
\mathbf{0} & \mathbf{0} & W^{2}\left|\mathbf{v}-\mathbf{v}^{*}\right|^{2}
\end{array}\right), \quad \mathbf{A}_{2}=\left(\begin{array}{ccc}
\mathbf{O} & \mathbf{O} & \mathbf{b}_{1}^{\top} \\
\mathbf{O} & \mathbf{O} & \mathbf{b}_{2}^{\top} \\
\mathbf{b}_{1} & \mathbf{b}_{2} & 0
\end{array}\right),
$$

where $\mathbf{0}=(0,0,0), \mathbf{O}$ is $3 \times 3$ zero matrix, and (note that $\mathbf{v}$ and $\mathbf{v}^{*}$ are row vectors)

$$
\begin{aligned}
& \mathbf{G}=\left(1-\left|\mathbf{v}^{*}\right|^{2}\right) \mathbf{I}_{3}+\left(\mathbf{v}^{*}\right)^{\top} \mathbf{v}^{*} \\
& \mathbf{H}=\left(1+\left|\mathbf{v}-\mathbf{v}^{*}\right|^{2}-\left|\mathbf{v}^{*}\right|^{2}\right) \mathbf{I}_{3}+\left(\mathbf{v}^{*}\right)^{\top} \mathbf{v}^{*}-\left(\mathbf{v}-\mathbf{v}^{*}\right)^{\top}\left(\mathbf{v}-\mathbf{v}^{*}\right), \\
& \mathbf{b}_{1}=\left(1-\left|\mathbf{v}^{*}\right|^{2}\right)\left(\mathbf{v}^{*}-\mathbf{v}\right)+\left(\left|\mathbf{v}^{*}\right|^{2}-\mathbf{v} \cdot \mathbf{v}^{*}\right) \mathbf{v}^{*}, \\
& \mathbf{b}_{2}=\left(1-|\mathbf{v}|^{2}\right)\left(\mathbf{v}-\mathbf{v}^{*}\right)+\left(|\mathbf{v}|^{2}-\mathbf{v} \cdot \mathbf{v}^{*}\right) \mathbf{v} .
\end{aligned}
$$

Then, it suffices to show that both $\mathbf{A}_{3}+\mathbf{A}_{2}$ and $\mathbf{A}_{3}-\mathbf{A}_{2}$ are positive semi-definite.

Note that $\mathbf{G}$ is symmetric, and its eigenvalues are $\left\{1-\left|\mathbf{v}^{*}\right|^{2}, 1-\left|\mathbf{v}^{*}\right|^{2}, 1\right\}$ and all positive, implying the positive definiteness of $\mathbf{G}$. Define a nonsingular matrix

$$
\mathbf{P}_{1}=\left(\begin{array}{ccc}
\mathbf{I}_{3} & \mathbf{O} & \mathbf{0}^{\top} \\
\mathbf{I}_{3} & \mathbf{I}_{3} & \mathbf{0}^{\top} \\
-\mathbf{b}_{1} \mathbf{G}^{-1} & \mathbf{0} & 1
\end{array}\right)
$$

where $-\mathbf{b}_{1} \mathbf{G}^{-1}=\mathbf{v}-\mathbf{v}^{*}$. Then

$$
\mathbf{P}_{1}\left(\mathbf{A}_{3}+\mathbf{A}_{2}\right) \mathbf{P}_{1}^{\top}=\left(\begin{array}{ccc}
\mathbf{G} & \mathbf{O} & \mathbf{0}^{\top} \\
\mathbf{O} & \mathbf{H}-\mathbf{G} & \mathbf{b}_{1}^{\top}+\mathbf{b}_{2}^{\top} \\
\mathbf{0} & \mathbf{b}_{1}+\mathbf{b}_{2} & W^{2}\left|\mathbf{v}-\mathbf{v}^{*}\right|^{2}-\mathbf{b}_{1} \mathbf{G}^{-1} \mathbf{b}_{1}^{\top}
\end{array}\right)
$$

where

$$
\mathbf{b}_{1}+\mathbf{b}_{2}=\left(\left|\mathbf{v}^{*}\right|^{2}-\mathbf{v} \cdot \mathbf{v}^{*}\right) \mathbf{v}+\left(|\mathbf{v}|^{2}-\mathbf{v} \cdot \mathbf{v}^{*}\right) \mathbf{v}^{*},
$$

and the matrix $\mathbf{H}-\mathbf{G}$ is symmetric and given by

$$
\mathbf{H}-\mathbf{G}=\left|\mathbf{v}-\mathbf{v}^{*}\right|^{2} \mathbf{I}_{3}-\left(\mathbf{v}-\mathbf{v}^{*}\right)^{\top}\left(\mathbf{v}-\mathbf{v}^{*}\right) .
$$

The eigenvalues of $\mathbf{H}-\mathbf{G}$ are $\left\{0,\left|\mathbf{v}-\mathbf{v}^{*}\right|^{2},\left|\mathbf{v}-\mathbf{v}^{*}\right|^{2}\right\}$, which are all nonnegative, implying that $\mathbf{H}-\mathbf{G}$ is positive semi-definite.

Now, we would like to show that $\mathbf{P}_{1}\left(\mathbf{A}_{3}+\mathbf{A}_{2}\right) \mathbf{P}_{1}^{\top}$ is positive semi-definite. Let us first consider two trivial cases:

- If $\mathbf{v}=\mathbf{v}^{*}$, then $\mathbf{P}_{1}\left(\mathbf{A}_{3}+\mathbf{A}_{2}\right) \mathbf{P}_{1}^{\top}=\mathbf{O}$, which is positive semi-definite.

- If $\mathbf{v}=\mathbf{0}$, then $\mathbf{b}_{1}=\mathbf{v}^{*}=-\mathbf{b}_{2}$ and $W^{2}\left|\mathbf{v}-\mathbf{v}^{*}\right|^{2}-\mathbf{b}_{1} \mathbf{G}^{-1} \mathbf{b}_{1}^{\top}=0$. In this case, $\mathbf{P}_{1}\left(\mathbf{A}_{3}+\right.$ $\left.\mathbf{A}_{2}\right) \mathbf{P}_{1}^{\top}=\operatorname{diag}\{\mathbf{G}, \mathbf{H}-\mathbf{G}, 0\}$, which is positive semi-definite.

In the following, we shall focus on the nontrivial case that $\mathbf{v} \neq \mathbf{v}^{*}$ and $\mathbf{v} \neq \mathbf{0}$. For any $\varepsilon>0$, we define

$$
\mathbf{Q}_{\varepsilon}=\left(\begin{array}{cc}
\mathbf{H}-\mathbf{G}+\varepsilon \mathbf{I}_{3} & \mathbf{b}_{1}^{\top}+\mathbf{b}_{2}^{\top} \\
\mathbf{b}_{1}+\mathbf{b}_{2} & W^{2}\left|\mathbf{v}-\mathbf{v}^{*}\right|^{2}-\mathbf{b}_{1} \mathbf{G}^{-1} \mathbf{b}_{1}^{\top}
\end{array}\right) .
$$


Some algebraic manipulations yield that

$$
\operatorname{det}\left(\mathbf{Q}_{\varepsilon}\right)=\frac{\varepsilon}{1-|\mathbf{v}|^{2}}\left(\varepsilon+\left|\mathbf{v}-\mathbf{v}^{*}\right|^{2}\right)\left(\varepsilon \Pi_{4}+|\mathbf{v}|^{2}\left|\mathbf{v}-\mathbf{v}^{*}\right|^{4}\right),
$$

where

$$
\Pi_{4}:=\left(1-|\mathbf{v}|^{2}\right)\left(\left(v_{1} v_{2}^{*}-v_{2} v_{1}^{*}\right)^{2}+\left(v_{2} v_{3}^{*}-v_{3} v_{2}^{*}\right)^{2}+\left(v_{3} v_{1}^{*}-v_{1} v_{3}^{*}\right)^{2}\right)+|\mathbf{v}|^{2}\left|\mathbf{v}-\mathbf{v}^{*}\right|^{2} .
$$

It is evident that $\Pi_{4} \geq|\mathbf{v}|^{2}\left|\mathbf{v}-\mathbf{v}^{*}\right|^{2}$. For any $\varepsilon>0$, the matrix $\mathbf{H}-\mathbf{G}+\varepsilon \mathbf{I}_{3}$ is positive definite, and when $\mathbf{v} \neq \mathbf{v}^{*}$ and $\mathbf{v} \neq \mathbf{0}$, it holds

$$
\operatorname{det}\left(\mathbf{Q}_{\varepsilon}\right) \geq \frac{\varepsilon}{1-|\mathbf{v}|^{2}}\left(\varepsilon+\left|\mathbf{v}-\mathbf{v}^{*}\right|^{2}\right)\left|\mathbf{v}-\mathbf{v}^{*}\right|^{2}|\mathbf{v}|^{2}\left(\varepsilon+\left|\mathbf{v}-\mathbf{v}^{*}\right|^{2}\right)>0
$$

This implies that the leading principal minors of $\mathbf{Q}_{\varepsilon}$ are all positive, and thus $\mathbf{Q}_{\varepsilon}$ is positive definite for any $\varepsilon>0, \mathbf{v} \neq \mathbf{v}^{*}$ and $\mathbf{v} \neq \mathbf{0}$. Taking the limit $\varepsilon \rightarrow 0$, we obtain that $\mathbf{Q}_{0}$ is positive semi-definite, which further yields that $\mathbf{P}_{1}\left(\mathbf{A}_{3}+\mathbf{A}_{2}\right) \mathbf{P}_{1}^{\top}=\operatorname{diag}\left\{\mathbf{G}, \mathbf{Q}_{0}\right\}$ is positive semi-definite, for the nontrivial case $\left(\mathbf{v} \neq \mathbf{v}^{*}\right.$ and $\left.\mathbf{v} \neq \mathbf{0}\right)$. In conclusion, for all the cases, $\mathbf{P}_{1}\left(\mathbf{A}_{3}+\mathbf{A}_{2}\right) \mathbf{P}_{1}^{\top}$ is positive semi-definite.

Because $\mathbf{A}_{3}+\mathbf{A}_{2}$ and $\mathbf{P}_{1}\left(\mathbf{A}_{3}+\mathbf{A}_{2}\right) \mathbf{P}_{1}^{\top}$ are congruent, $\mathbf{A}_{3}+\mathbf{A}_{2}$ is positive semi-definite. Similar arguments imply that $\mathbf{A}_{3}-\mathbf{A}_{2}$ is also positive semi-definite. Hence

$$
\Pi_{3} \pm \Pi_{2}=\frac{1}{2} \mathbf{Z}^{\top}\left(\mathbf{A}_{3} \pm \mathbf{A}_{2}\right) \mathbf{Z} \geq 0
$$

which yields $\Pi_{1} \geq \Pi_{3} \geq\left|\Pi_{2}\right|$. The proof is complete.

We also need the following technical inequality (20), which was constructed in [39], to handle the effect of flux in numerical PCP analysis.

Lemma 3.6. If $\mathbf{U} \in \mathcal{G}$, then for any $\theta \in[-1,1]$, any $\mathbf{B}^{*} \in \mathbb{R}^{3}$ and any $\mathbf{v}^{*} \in \mathbb{B}_{1}(\mathbf{0})$, it holds

$$
\left(\mathbf{U}+\theta \mathbf{F}_{i}(\mathbf{U})\right) \cdot \boldsymbol{\xi}^{*}+p_{m}^{*}+\theta\left(v_{i}^{*} p_{m}^{*}-B_{i}\left(\mathbf{v}^{*} \cdot \mathbf{B}^{*}\right)\right) \geq 0,
$$

where $i \in\{1,2, \cdots, d\}$, and $\boldsymbol{\xi}^{*}$ and $p_{m}^{*}$ are defined in (15) and (16), respectively.

For any vector $\mathbf{n}=\left(n_{1}, \cdots, n_{d}\right) \in \mathbb{R}^{d}$, we define the inner products

$$
\langle\mathbf{n}, \mathbf{v}\rangle:=\sum_{k=1}^{d} n_{k} v_{k}, \quad\langle\mathbf{n}, \mathbf{B}\rangle:=\sum_{k=1}^{d} n_{k} B_{k}, \quad\langle\mathbf{n}, \mathbf{F}\rangle:=\sum_{k=1}^{d} n_{k} \mathbf{F}_{k}
$$

which will be frequently used in this paper. Then we can generalize Lemma 3.6.

Lemma 3.7. If $\mathbf{U} \in \mathcal{G}$, then for any $\theta \in[-1,1]$, any $\mathbf{B}^{*} \in \mathbb{R}^{3}$, any $\mathbf{v}^{*} \in \mathbb{B}_{1}(\mathbf{0})$, and any unit vector $\mathbf{n} \in \mathbb{R}^{d}$, it holds

$$
(\mathbf{U}+\theta\langle\mathbf{n}, \mathbf{F}(\mathbf{U})\rangle) \cdot \boldsymbol{\xi}^{*}+p_{m}^{*}+\theta\left(\left\langle\mathbf{n}, \mathbf{v}^{*}\right\rangle p_{m}^{*}-\langle\mathbf{n}, \mathbf{B}\rangle\left(\mathbf{v}^{*} \cdot \mathbf{B}^{*}\right)\right) \geq 0 .
$$

Proof. This can be proven by using Lemma 3.6 and the rotational invariance of the RMHD system. The proof is omitted.

4. Provably PCP DG Schemes. In this section, we construct PCP high-order DG schemes for the multidimensional RMHD based on the symmetrizable form (9). For the sake of clarity, we shall mainly focus on the $2 \mathrm{D}$ case $(d=2)$, keeping in mind that our PCP methods and analyses are also extendable to the $3 \mathrm{D}$ case $(d=3)$. 
4.1. Outline of the PCP schemes. Assume that the $2 \mathrm{D}$ spatial domain $\Omega$ is partitioned into a mesh $\mathcal{T}_{h}$, which may be unstructured and consists of polygonal cells. The time interval is also divided into the mesh $\left\{t_{0}=0, t_{n+1}=t_{n}+\Delta t_{n}, 0 \leq n<N_{t}\right\}$ with the time step-size $\Delta t_{n}$ determined by some CFL condition. Throughout this section, the lower-case letter $k$ is used to denote the DG polynomial degree, while the capital letter $K$ always represents a cell in $\mathcal{T}_{h}$.

Let $\mathbf{x} \in \mathbb{R}^{d}$ denote the spatial coordinate vector. We define the locally divergence-free DG finite element space [17]

$$
\mathbb{W}_{h}^{k}=\left\{\mathbf{u}=\left(u_{1}, \cdots, u_{8}\right)^{\top}:\left.u_{\ell}\right|_{K} \in \mathbb{P}^{k}(K), \forall \ell,\left.\sum_{i=1}^{d} \frac{\partial u_{4+i}}{\partial x_{i}}\right|_{K}=0, \forall K \in \mathcal{T}_{h}\right\},
$$

where $\mathbb{P}^{k}(K)$ denotes the space of polynomials, in cell $K$, of total degree up to $k$. To define the PCP DG schemes, we also introduce the following two subsets of $\mathbb{W}_{h}^{k}$ :

$$
\begin{aligned}
& \overline{\mathbb{G}}_{h}^{k}:=\left\{\mathbf{u} \in \mathbb{W}_{h}^{k}: \frac{1}{|K|} \int_{K} \mathbf{u}(\mathbf{x}) d \mathbf{x} \in \mathcal{G}, \quad \forall K \in \mathcal{T}_{h}\right\}, \\
& \mathbb{G}_{h}^{k}:=\left\{\mathbf{u} \in \overline{\mathbb{G}}_{h}^{k}:\left.\mathbf{u}\right|_{K}(\mathbf{x}) \in \mathcal{G}, \quad \forall \mathbf{x} \in \mathbb{S}_{K}, \forall K \in \mathcal{T}_{h}\right\},
\end{aligned}
$$

where $|K|$ denotes the area of the cell $K$, and $\mathbb{S}_{K}$ denotes the set of some critical points in $K$ which will be specified later.

Definition 4.1. A DG scheme is defined to be PCP if its solutions always stay in $\mathbb{G}_{h}^{k}$. For clarity, if a DG scheme preserves the numerical solutions in $\overline{\mathbb{G}}_{h}^{k}$, then we say it satisfies a "weak" PCP property.

LEMmA 4.2. The sets $\overline{\mathbb{G}}_{h}^{k}$ and $\mathbb{G}_{h}^{k}$ are both convex. In addition, for any vector function $\mathbf{u} \in$ $\left[L^{2}(\Omega)\right]^{8}$ satisfying $\mathbf{u}(\mathbf{x}) \in \mathcal{G}, \forall \mathbf{x} \in \Omega$, we have $\mathbf{P}_{w}(\mathbf{u}) \in \overline{\mathbb{G}}_{h}^{k}$, where $\mathbf{P}_{w}$ denoting the $L^{2}$-projection into $\mathbb{W}_{h}^{k}$.

Proof. These conclusions directly follow from that $\mathcal{G}=\mathcal{G}_{1}$ is a convex set, which is implied by Lemmas 3.1 and 3.4. The proof is omitted.

We aim at constructing PCP high-order accurate DG schemes that always preserve the DG solution function $\mathbf{U}_{h}(\mathbf{x}, t)$ in the set $\mathbb{G}_{h}^{k}$ for all $t \in\left\{t_{n}: 0 \leq n \leq N_{t}\right\}$. This goal will be achieved by following three steps:

1. First, we will seek in Sect. 4.2 a suitable spatial discretization of symmetrizable RMHD system (9), such that the resulting discrete equation, which can be put in ODE form as $\frac{d}{d t} \mathbf{U}_{h}=\mathbf{L}_{h}\left(\mathbf{U}_{h}\right)$, satisfies the "weak" PCP property

$$
\text { if } \mathbf{U}_{h} \in \mathbb{G}_{h}^{k}, \quad \text { then } \mathbf{U}_{h}+\Delta t \mathbf{L}_{h}\left(\mathbf{U}_{h}\right) \in \overline{\mathbb{G}}_{h}^{k},
$$

under some CFL condition on $\Delta t$. The property (25) is very important. It is extremely nontrivial to find a DG discretization for the RMHD that can be proven to satisfy (25). Some traditional methods including standard DG schemes for the conservative RMHD system (1) do not satisfy (25).

2. Then, we further discretize the ODE system $\frac{d}{d t} \mathbf{U}_{h}=\mathbf{L}_{h}\left(\mathbf{U}_{h}\right)$ in time using a strong-stabilitypreserving (SSP) explicit Runge-Kutta method [12].

3. Finally, a local scaling PCP limiting procedure, which will be introduced in Sect. 4.3, is applied to the intermediate solutions of the Runge-Kutta discretization. This procedure corresponds to an operator $\boldsymbol{\Pi}_{h}: \overline{\mathbb{G}}_{h}^{k} \longrightarrow \mathbb{G}_{h}^{k}$, which maps the numerical solutions from $\overline{\mathbb{G}}_{h}^{k}$ to $\mathbb{G}_{h}^{k}$ and satisfies

$$
\frac{1}{|K|} \int_{K} \boldsymbol{\Pi}_{h}(\mathbf{u}) d \mathbf{x}=\frac{1}{|K|} \int_{K} \mathbf{u} d \mathbf{x}, \quad \forall K \in \mathcal{T}_{h}, \quad \forall \mathbf{u} \in \overline{\mathbb{G}}_{h}^{k} .
$$

The PCP limiter is required only for high-order DG methods with $k \geq 1$; for the first-order DG method $(k=0), \boldsymbol{\Pi}_{h}$ becomes the identity operator. 
Let $\mathbf{U}_{h}^{n}$ denote the numerical solution at time $t=t_{n}$. The resulting fully discrete PCP DG methods, with a $N_{r}$-stage SSP Runge-Kutta method, can be written in the following form:

- Set $\mathbf{U}_{h}^{0}=\boldsymbol{\Pi}_{h} \mathbf{P}_{w}(\mathbf{U}(\mathbf{x}, 0))$;

- For $n=0, \ldots, N_{t}-1$, compute $\mathbf{U}_{h}^{n+1}$ as follows:

(i) set $\mathbf{U}_{h}^{(0)}=\mathbf{U}_{h}^{n}$;

(ii) for $i=1, \ldots, N_{r}$ compute the intermediate solutions:

$$
\mathbf{U}_{h}^{(i)}=\boldsymbol{\Pi}_{h}\left\{\sum_{\ell=0}^{i-1}\left[\alpha_{i \ell}\left(\mathbf{U}_{h}^{(\ell)}+\beta_{i \ell} \Delta t_{n} \mathbf{L}_{h}\left(\mathbf{U}_{h}^{(\ell)}\right)\right)\right]\right\} ;
$$

(iii) set $\mathbf{U}_{h}^{n+1}=\mathbf{U}_{h}^{\left(N_{r}\right)}$;

where the SSP Runge-Kutta method has been written into a convex combination of the forward Euler method, and the associated parameters $\alpha_{i \ell}$ and $\beta_{i \ell}$ are all non-negative and satisfy $\sum_{\ell=0}^{i-1} \alpha_{i \ell}=1$. Some SSP Runge-Kutta methods can be found in [12, 28], e.g., a commonly-used three-stage thirdorder version is given by

$$
\begin{aligned}
& \alpha_{10}=1, \quad \alpha_{20}=3 / 4, \quad \alpha_{21}=1 / 4, \quad \alpha_{30}=1 / 3, \quad \alpha_{31}=0, \quad \alpha_{32}=2 / 3, \\
& \beta_{10}=1, \quad \beta_{20}=0, \quad \beta_{21}=1, \quad \beta_{30}=0, \quad \beta_{31}=0, \quad \beta_{32}=1 .
\end{aligned}
$$

Remark 4.3. At each Runge-Kutta stage, the PCP property of the above fully discrete DG schemes is enforced by the operator $\Pi_{h}$, which can only act on functions in $\overline{\mathbb{G}}_{h}^{k}$. That is, we require the convex combination $\sum_{\ell=0}^{i-1}\left[\alpha_{i \ell}\left(\mathbf{U}_{h}^{(\ell)}+\beta_{i \ell} \Delta t_{n} \mathbf{L}_{h}\left(\mathbf{U}_{h}^{(\ell)}\right)\right)\right] \in \overline{\mathbb{G}}_{h}^{k}$, which is guaranteed by the weak PCP property (25) and the convexity of $\overline{\mathbb{G}}_{h}^{k}$. On the other hand, the PCP limiting operator $\Pi_{h}$ enforces $\mathbf{U}_{h}^{(\ell)} \in \mathbb{G}_{h}^{k}, 0 \leq \ell<i$, which provides the condition required by the weak PCP property (25) for the next Runge-Kutta stage evolution. Therefore, the weak PCP property (25) and the PCP limiting operator $\boldsymbol{\Pi}_{h}$ are two key ingredients of the proposed PCP schemes.

In what follows, we shall describe in detail the operators $\mathbf{L}_{h}$ and $\boldsymbol{\Pi}_{h}$, and also specify the point set $\mathbb{S}_{K}$ in the definition (24) of $\mathbb{G}_{h}^{k}$. We will prove the weak PCP property (25) of the DG spatial discretization in Theorem 4.4 and the PCP property of the fully discrete DG schemes in Theorems 4.9 and 4.10 .

4.2. The operator $\mathbf{L}_{h}$ and the weak PCP property. We now derive a suitable spatial discretization such that the resulting operator $\mathbf{L}_{h}$ satisfies the weak PCP property (25). Following our previous work on the ideal non-relativistic MHD [35, 37], we consider the following locally divergencefree DG methods for the symmetrizable RMHD system (9):

$$
\begin{aligned}
& \frac{d}{d t} \int_{K} \mathbf{U}_{h}(\mathbf{x}, t) \cdot \mathbf{u} d \mathbf{x}=\int_{K} \mathbf{F}\left(\mathbf{U}_{h}\right) \cdot \nabla \mathbf{u} d \mathbf{x} \\
& -\sum_{\mathscr{E} \in \partial K} \int_{\mathscr{E}} \mathbf{u}^{\operatorname{int}(K)} \cdot\left\{\hat{\mathbf{F}}\left(\mathbf{U}_{h}^{\operatorname{int}(K)}, \mathbf{U}_{h}^{\operatorname{ext}(K)} ; \mathbf{n}_{\mathscr{E}, K}\right)\right. \\
& \left.\quad+\left[\frac{1}{2}\left\langle\mathbf{n}_{\mathscr{E}, K}, \mathbf{B}_{h}^{\operatorname{ext}(K)}-\mathbf{B}_{h}^{\operatorname{int}(K)}\right\rangle \mathbf{S}\left(\mathbf{U}_{h}^{\operatorname{int}(K)}\right)\right]\right\} d s, \quad \forall \mathbf{u} \in \mathbb{W}_{h}^{k},
\end{aligned}
$$

where $\partial K$ denotes the boundary of the cell $K ; \mathbf{n}_{\mathscr{E}, K}$ is the outward unit normal to the edge $\mathscr{E}$ of $K$; the inner product $\langle\cdot, \cdot\rangle$ is defined in $(21)$; the superscripts "int $(K)$ " and "ext $(K)$ " indicate that the associated limits of $\mathbf{U}_{h}(\mathbf{x})$ at the cell interfaces are taken from the interior and exterior of $K$, respectively. In (29), $\hat{\mathbf{F}}$ denotes the numerical flux, which we take as the global Lax-Friedrichs flux

$$
\begin{aligned}
\hat{\mathbf{F}} & \left(\mathbf{U}_{h}^{\operatorname{int}(K)}, \mathbf{U}_{h}^{\operatorname{ext}(K)} ; \mathbf{n}_{\mathscr{E}, K}\right) \\
& =\frac{1}{2}\left(\left\langle\mathbf{n}_{\mathscr{E}, K}, \mathbf{F}\left(\mathbf{U}_{h}^{\operatorname{int}(K)}\right)+\mathbf{F}\left(\mathbf{U}_{h}^{\operatorname{ext}(K)}\right)\right\rangle-a\left(\mathbf{U}_{h}^{\operatorname{ext}(K)}-\mathbf{U}_{h}^{\operatorname{int}(K)}\right)\right),
\end{aligned}
$$


where the numerical viscosity parameter $a$ is taken as the speed of light $c=1$, a simple upper bound of all wave speeds. The term inside the square bracket in (29) is derived from a suitable discretization of the source term in the symmetrizable RMHD system (9), where the locally divergence-free property of $\mathbf{B}_{h}$ has been taken into account. This term is proportional to the jump of the normal magnetic component across cell interface, which is zero for the exact solution and is very small (at the level of truncation error) for numerical solutions. The inclusion of this term is crucial for achieving the property (25), as demonstrated by our theoretical analysis later.

Of course, we have to replace the boundary and element integrals at the right-hand side of (29) by some quadrature rules of sufficiently high-order accuracy (specifically, the algebraic degree of accuracy should be at least $2 k$ ). For example, we can approximate the boundary integral by the Gauss quadrature with $Q=k+1$ points:

$$
\begin{aligned}
\int_{\mathscr{E}} \mathbf{u}^{\operatorname{int}(K)} \cdot\left[\hat{\mathbf{F}}\left(\mathbf{U}_{h}^{\operatorname{int}(K)}, \mathbf{U}_{h}^{\operatorname{ext}(K)} ; \mathbf{n}_{\mathscr{E}, K}\right)+\frac{1}{2}\left\langle\mathbf{n}_{\mathscr{E}, K}, \mathbf{B}_{h}^{\operatorname{ext}(K)}-\mathbf{B}_{h}^{\operatorname{int}(K)}\right\rangle \mathbf{S}\left(\mathbf{U}_{h}^{\operatorname{int}(K)}\right)\right] d s \\
\approx|\mathscr{E}| \sum_{q=1}^{Q} \omega_{q} \mathbf{u}^{\operatorname{int}(K)}\left(\mathbf{x}_{\mathscr{E}}^{(q)}\right) \cdot\left[\hat{\mathbf{F}}\left(\mathbf{U}_{h}^{\operatorname{int}(K)}\left(\mathbf{x}_{\mathscr{E}}^{(q)}, t\right), \mathbf{U}_{h}^{\operatorname{ext}(K)}\left(\mathbf{x}_{\mathscr{E}}^{(q)}, t\right) ; \mathbf{n}_{\mathscr{E}, K}\right)\right. \\
\left.\quad+\frac{1}{2}\left\langle\mathbf{n}_{\mathscr{E}, K}, \mathbf{B}_{h}^{\operatorname{ext}(K)}\left(\mathbf{x}_{\mathscr{E}}^{(q)}, t\right)-\mathbf{B}_{h}^{\operatorname{int}(K)}\left(\mathbf{x}_{\mathscr{E}}^{(q)}, t\right)\right\rangle \mathbf{S}\left(\mathbf{U}_{h}^{\operatorname{int}(K)}\left(\mathbf{x}_{\mathscr{E}}^{(q)}, t\right)\right)\right],
\end{aligned}
$$

where $|\mathscr{E}|$ denotes the length of the edge $\mathscr{E},\left\{\mathbf{x}_{\mathscr{E}}^{(q)}\right\}_{1 \leq q \leq Q}$ are the quadrature points on $\mathscr{E}$, and $\left\{\omega_{q}\right\}_{1 \leq q \leq Q}$ are the associated weights with $\sum_{q=1}^{Q} \omega_{q}=1$. The element integral $\int_{K} \mathbf{F}\left(\mathbf{U}_{h}\right) \cdot \nabla \mathbf{u} d \mathbf{x}$ can also be approximated by some 2D quadrature $|K| \sum_{q=1}^{\breve{Q}} \breve{\omega}_{q} \mathbf{F}\left(\mathbf{U}_{h}\left(\breve{\mathbf{x}}_{K}^{(q)}, t\right)\right) \cdot \nabla \mathbf{u}\left(\breve{\mathbf{x}}_{K}^{(q)}\right)$, where $\breve{\mathbf{x}}_{K}^{(q)}$ and $\breve{\omega}_{q}$ denote the quadrature points and weights, respectively.

Thus, we finally obtain the weak formulation:

$$
\frac{d}{d t} \int_{K} \mathbf{U}_{h} \cdot \mathbf{u} d \mathbf{x}=\mathcal{J}_{K}\left(\mathbf{U}_{h}, \mathbf{u}\right), \quad \forall \mathbf{u} \in \mathbb{W}_{h}^{k}
$$

where $\mathcal{J}_{K}\left(\mathbf{U}_{h}, \mathbf{u}\right)=\sum_{i=1}^{3} \mathcal{J}_{K}^{(i)}\left(\mathbf{U}_{h}, \mathbf{u}\right)$ with

$$
\begin{aligned}
\mathcal{J}_{K}^{(1)} & =-\sum_{\mathscr{E} \in \partial K}\left\{|\mathscr{E}| \sum_{q=1}^{Q} \omega_{q} \hat{\mathbf{F}}\left(\mathbf{U}_{h}^{\operatorname{int}(K)}\left(\mathbf{x}_{\mathscr{E}}^{(q)}\right), \mathbf{U}_{h}^{\operatorname{ext}(K)}\left(\mathbf{x}_{\mathscr{E}}^{(q)}\right) ; \mathbf{n}_{\mathscr{E}, K}\right) \cdot \mathbf{u}^{\operatorname{int}(K)}\left(\mathbf{x}_{\mathscr{E}}^{(q)}\right)\right\} \\
\mathcal{J}_{K}^{(2)} & =-\frac{1}{2} \sum_{\mathscr{E} \in \partial K}\left\{|\mathscr{E}| \sum_{q=1}^{Q} \omega_{q}\left\langle\mathbf{n}_{\mathscr{E}, K}, \mathbf{B}_{h}^{\operatorname{ext}(K)}\left(\mathbf{x}_{\mathscr{E}}^{(q)}\right)-\mathbf{B}_{h}^{\operatorname{int}(K)}\left(\mathbf{x}_{\mathscr{E}}^{(q)}\right)\right\rangle \mathbf{S}\left(\mathbf{U}_{h}^{\operatorname{int}(K)}\left(\mathbf{x}_{\mathscr{E}}^{(q)}\right)\right) \cdot \mathbf{u}^{\operatorname{int}(K)}\left(\mathbf{x}_{\mathscr{E}}^{(q)}\right)\right\} \\
\mathcal{J}_{K}^{(3)} & =|K| \sum_{q=1}^{\breve{Q}} \breve{\omega}_{q} \mathbf{F}\left(\mathbf{U}_{h}\left(\breve{\mathbf{x}}_{K}^{(q)}\right)\right) \cdot \nabla \mathbf{u}\left(\breve{\mathbf{x}}_{K}^{(q)}\right)
\end{aligned}
$$

and for notational convenience, the $t$ dependence of all quantities is suppressed hereafter, unless confusion arises otherwise. As the standard DG methods (cf. $[5,4]$ ), the weak form (31) can be rewritten in the ODE form as

$$
\frac{d}{d t} \mathbf{U}_{h}=\mathbf{L}_{h}\left(\mathbf{U}_{h}\right)
$$

after choosing a suitable basis of $\mathbb{W}_{h}^{k}$ and representing $\mathbf{U}_{h}$ as a linear combination of the basis functions; see $[5,4]$ for details. Note that the corresponding cell average, denoted by $\overline{\mathbf{U}}_{K}:=\frac{1}{|K|} \int_{K} \mathbf{U}_{h} d \mathbf{x}$, satisfies the following time evolution equation

$$
\frac{d}{d t} \overline{\mathbf{U}}_{K}=\widetilde{\mathcal{J}}_{K}\left(\mathbf{U}_{h}\right), \quad \forall K \in \mathcal{T}_{h},
$$


where $\widetilde{\mathcal{J}}_{K}\left(\mathbf{U}_{h}\right)=\widetilde{\mathcal{J}}_{K}^{(1)}\left(\mathbf{U}_{h}\right)+\widetilde{\mathcal{J}}_{K}^{(2)}\left(\mathbf{U}_{h}\right)$ with

$$
\begin{aligned}
& \widetilde{\mathcal{J}}_{K}^{(1)}\left(\mathbf{U}_{h}\right)=-\frac{1}{|K|} \sum_{\mathscr{E} \in \partial K}\left\{|\mathscr{E}| \sum_{q=1}^{Q} \omega_{q} \hat{\mathbf{F}}\left(\mathbf{U}_{h}^{\operatorname{int}(K)}\left(\mathbf{x}_{\mathscr{E}}^{(q)}\right), \mathbf{U}_{h}^{\operatorname{ext}(K)}\left(\mathbf{x}_{\mathscr{E}}^{(q)}\right) ; \mathbf{n}_{\mathscr{E}, K}\right)\right\} \\
& \widetilde{\mathcal{J}}_{K}^{(2)}\left(\mathbf{U}_{h}\right)=-\frac{1}{2|K|} \sum_{\mathscr{E} \in \partial K}\left\{|\mathscr{E}| \sum_{q=1}^{Q} \omega_{q}\left\langle\mathbf{n}_{\mathscr{E}, K}, \mathbf{B}_{h}^{\operatorname{ext}(K)}\left(\mathbf{x}_{\mathscr{E}}^{(q)}\right)-\mathbf{B}_{h}^{\operatorname{int}(K)}\left(\mathbf{x}_{\mathscr{E}}^{(q)}\right)\right\rangle \mathbf{S}\left(\mathbf{U}_{h}^{\operatorname{int}(K)}\left(\mathbf{x}_{\mathscr{E}}^{(q)}\right)\right)\right\}
\end{aligned}
$$

We are now in a position to rigorously prove that the above DG spatial discretization satisfies the weak PCP property (25). To this end, we first need to specify the point set $\mathbb{S}_{K}$ in the definition (24) of $\mathbb{G}_{h}^{k}$. Assume that there exists a special 2D quadrature on each cell $K \in \mathcal{T}_{h}$ satisfying:

(i) The quadrature rule is with positive weights and exact for integrals of polynomials of degree up to $k$ on the cell $K$.

(ii) The set of the quadrature points, denoted by $\widehat{\mathbb{S}}_{K}$, must include all the Gauss quadrature points $\mathbf{x}_{\mathscr{E}}^{(q)}, q=1, \ldots, Q$, on all the edge $\mathscr{E} \in \partial K$.

In other words, we would like to have a special quadrature such that

$$
\frac{1}{|K|} \int_{K} u(\mathbf{x}) d \mathbf{x}=\sum_{\mathscr{E} \in \partial K} \sum_{q=1}^{Q} \varpi_{\mathscr{E}}^{(q)} u\left(\mathbf{x}_{\mathscr{E}}^{(q)}\right)+\sum_{q=1}^{\widetilde{Q}} \widetilde{\varpi}_{q} u\left(\widetilde{\mathbf{x}}_{K}^{(q)}\right), \quad \forall u \in \mathbb{P}^{k}(K),
$$

where $\left\{\widetilde{\mathbf{x}}_{K}^{(q)}\right\}$ are the other (possible) quadrature points in $K$, and the quadrature weights $\varpi_{\mathscr{E}}^{(q)}$ and $\widetilde{\varpi}_{q}$ are positive and satisfy $\sum_{\mathscr{E} \in \partial K} \sum_{q=1}^{Q} \varpi_{\mathscr{E}}^{(q)}+\sum_{q=1}^{\widetilde{Q}} \widetilde{\varpi}_{q}=1$. For rectangular cells, such a quadrature was constructed in $[46,47]$ by tensor products of Gauss quadrature and Gauss-Lobatto quadrature. For triangular cells and more general polygons, see $[48,32,8]$ for how to construct such a special quadrature. We remark that this special quadrature is not used for computing any integrals, but only used in the following theoretical PCP analysis and the PCP limiter presented later.

Given this special quadrature, we define the point set $\mathbb{S}_{K}$ required in (24) as

$$
\mathbb{S}_{K}=\widehat{\mathbb{S}}_{K} \cup \breve{S}_{K}
$$

where $\breve{\mathbb{S}}_{K}:=\left\{\breve{\mathbf{x}}_{K}^{(q)}, 1 \leq q \leq \breve{Q}\right\}$ are the quadrature points involved in $\mathcal{J}_{K}^{(3)}$. The inclusion of $\breve{\mathbb{S}}_{K}$ means that we require $\mathbf{U}_{h}\left(\breve{\mathbf{x}}_{K}^{(q)}\right) \in \mathcal{G}$. This special requirement does not appear in the non-relativistic case; it is used here to ensure the existence and uniqueness of the physically admissible solution of the nonlinear equation (6) and thus obtaining the physical primitive variables from $\mathbf{U}_{h}\left(\breve{\mathbf{x}}_{K}^{(q)}\right)$ by (7), so as to successfully compute $\mathbf{F}\left(\mathbf{U}_{h}\left(\breve{\mathbf{x}}_{K}^{(q)}\right)\right)$ in $\mathcal{J}_{K}^{(3)}$. Such a consideration is due to that the flux $\mathbf{F}(\mathbf{U})$ and source $\mathbf{S}(\mathbf{U})$ cannot be explicitly formulated in terms of $\mathbf{U}$ for the RMHD and thus must be computed using the corresponding primitive variables. Note that the edge quadrature points $\left\{\mathbf{x}_{\mathscr{E}}^{(q)}\right\}$, involved in $\mathcal{J}_{K}^{(1)}$ and $\mathcal{J}_{K}^{(2)}$, are already included in $\widehat{\mathbb{S}}_{K}$.

Based on the point set $\mathbb{S}_{K}$ defined above, we establish the weak PCP property (25) for the high-order semi-discrete DG scheme (32) as follows.

Theorem 4.4. Let $\mathbb{G}_{K}^{h}$ be the set defined by (24) with $\mathbb{S}_{K}$ specified in (35). Then, the weak PCP property (25) holds under the following CFL type condition

$$
\Delta t \frac{|\mathscr{E}|}{|K|}\left(a+\sigma_{K, \mathscr{E}, q}\left(\mathbf{U}_{h}\right)\right)<\frac{\varpi_{\mathscr{E}}^{(q)}}{\omega_{q}}, \quad 1 \leq q \leq Q, \forall \mathscr{E} \in \partial K, \forall K \in \mathcal{T}_{h},
$$

where $\sigma_{K, \mathscr{E}, q}\left(\mathbf{U}_{h}\right):=\frac{1}{2}\left|\left\langle\mathbf{n}_{\mathscr{E}, K}, \mathbf{B}_{\mathscr{E}, q}^{\operatorname{int}(K)}-\mathbf{B}_{\mathscr{E}, q}^{\operatorname{ext}(K)}\right\rangle\right| / \sqrt{\rho_{\mathscr{E}, q}^{\operatorname{int}(K)} H_{\mathscr{E}, q}^{\operatorname{int}(K)}}$ with the shortened notations $\mathbf{U}_{\mathscr{E}, q}^{\operatorname{int}(K)}:=\mathbf{U}_{h}^{\operatorname{int}(K)}\left(\mathbf{x}_{\mathscr{E}}^{(q)}\right)$ and $\mathbf{U}_{\mathscr{E}, q}^{\operatorname{ext}(K)}:=\mathbf{U}_{h}^{\operatorname{ext}(K)}\left(\mathbf{x}_{\mathscr{E}}^{(q)}\right)$. 
Proof. In order to prove $\mathbf{U}_{h}+\Delta t \mathbf{L}_{h}\left(\mathbf{U}_{h}\right) \in \overline{\mathbb{G}}_{h}^{k}$ in (25), it suffices to show

$$
\overline{\mathbf{U}}_{K}^{\Delta t}:=\overline{\mathbf{U}}_{K}+\Delta t \widetilde{\mathcal{J}}_{K}\left(\mathbf{U}_{h}\right) \in \mathcal{G}, \quad \forall K \in \mathcal{T}_{h},
$$

under the CFL type condition (45) and the condition that $\mathbf{U}_{h} \in \mathbb{G}_{h}^{k}$. Substituting the formula of the numerical flux (30) into $\widetilde{\mathcal{J}}_{K}^{(1)}\left(\mathbf{U}_{h}\right)$, we reformulate $\widetilde{\mathcal{J}}_{K}^{(1)}\left(\mathbf{U}_{h}\right)$ and decompose it into three parts:

$$
\begin{aligned}
\widetilde{\mathcal{J}}_{K}^{(1)}\left(\mathbf{U}_{h}\right)= & \frac{a}{2|K|} \sum_{\mathscr{E} \in \partial K}\left[|\mathscr{E}| \sum_{q=1}^{Q} \omega_{q}\left(\mathbf{U}_{\mathscr{E}, q}^{\operatorname{int}(K)}-\frac{1}{a}\left\langle\mathbf{n}_{\mathscr{E}, K}, \mathbf{F}\left(\mathbf{U}_{\mathscr{E}, q}^{\operatorname{int}(K)}\right)\right\rangle\right)\right] \\
& +\frac{a}{2|K|} \sum_{\mathscr{E} \in \partial K}\left[|\mathscr{E}| \sum_{q=1}^{Q} \omega_{q}\left(\mathbf{U}_{\mathscr{E}, q}^{\operatorname{ext}(K)}-\frac{1}{a}\left\langle\mathbf{n}_{\mathscr{E}, K}, \mathbf{F}\left(\mathbf{U}_{\mathscr{E}, q}^{\operatorname{ext}(K)}\right)\right\rangle\right)\right] \\
& -\frac{a}{|K|} \sum_{\mathscr{E} \in \partial K}\left(|\mathscr{E}| \sum_{q=1}^{Q} \omega_{q} \mathbf{U}_{\mathscr{E}, q}^{\operatorname{int}(K)}\right)=: \widetilde{\mathcal{J}}_{K}^{(1,1)}+\widetilde{\mathcal{J}}_{K}^{(1,2)}+\widetilde{\mathcal{J}}_{K}^{(1,3)} .
\end{aligned}
$$

Then $\overline{\mathbf{U}}_{K}^{\Delta t}$ can be rewritten as

$$
\overline{\mathbf{U}}_{K}^{\Delta t}=\boldsymbol{\Xi}_{1}+\boldsymbol{\Xi}_{2}+\boldsymbol{\Xi}_{3}+\boldsymbol{\Xi}_{4}
$$

with, $\boldsymbol{\Xi}_{i}:=\Delta t \widetilde{\mathcal{J}}_{K}^{(1, i)}, i=1,2, \boldsymbol{\Xi}_{3}:=\overline{\mathbf{U}}_{K}+\Delta t \widetilde{\mathcal{J}}_{K}^{(1,3)}$ and

$$
\boldsymbol{\Xi}_{4}:=\Delta t \widetilde{\mathcal{J}}_{K}^{(2)}=\frac{\Delta t}{2|K|} \sum_{\mathscr{E} \in \partial K}\left[|\mathscr{E}| \sum_{q=1}^{Q} \omega_{q}\left\langle\mathbf{n}_{\mathscr{E}, K}, \mathbf{B}_{\mathscr{E}, q}^{\operatorname{int}(K)}-\mathbf{B}_{\mathscr{E}, q}^{\operatorname{ext}(K)}\right\rangle \mathbf{S}\left(\mathbf{U}_{\mathscr{E}, q}^{\operatorname{int}(K)}\right)\right]
$$

Since $\mathcal{G}=\mathcal{G}_{2}$ as shown in Lemma 3.2, it remains to prove $\overline{\mathbf{U}}_{K}^{\Delta t} \in \mathcal{G}_{2}, \forall K \in \mathcal{T}_{h}$.

We fist show $\bar{D}_{K}^{\Delta t}>0$. Because $\mathbf{U}_{h} \in \mathbb{G}_{h}^{k}$ and $\widehat{\mathbb{S}}_{K} \subset \mathbb{S}_{K}$, we have $\mathbf{U}_{\mathscr{E}, q}^{\operatorname{int}(K)} \in \mathcal{G}$ and $\mathbf{U}_{\mathscr{E}, q}^{\operatorname{ext}(K)} \in \mathcal{G}$ for all $1 \leq q \leq Q, \mathscr{E} \in \partial K$ and $K \in \mathcal{T}_{h}$. Note that the first component of $\mathbf{U}_{\mathscr{E}, q}^{\operatorname{int}(K)}-\frac{1}{a}\left\langle\mathbf{n}_{\mathscr{E}, K}, \mathbf{F}\left(\mathbf{U}_{\mathscr{E}, q}^{\operatorname{int}(K)}\right)\right\rangle$ equals $D_{\mathscr{E}, q}^{\operatorname{int}(K)}\left(1-\frac{1}{a}\left\langle\mathbf{n}_{\mathscr{E}, K}, \mathbf{v}_{\mathscr{E}, q}^{\operatorname{int}(K)}\right\rangle\right) \geq D_{\mathscr{E}, q}^{\operatorname{int}(K)}\left(1-\frac{1}{a}\left|\mathbf{v}_{\mathscr{E}, q}^{\operatorname{int}(K)}\right|\right)>0$, which implies that the first component of $\boldsymbol{\Xi}_{1}$ is positive. Similarly, we know that the first component of $\boldsymbol{\Xi}_{2}$ is also positive. Notice that the first component of $\boldsymbol{\Xi}_{4}$ is zero. Therefore, the first component of $\overline{\mathbf{U}}_{K}^{\Delta t}$ is larger than that of $\boldsymbol{\Xi}_{3}$. It gives

$$
\begin{aligned}
\bar{D}_{K}^{\Delta t} & >\bar{D}_{K}-\frac{a \Delta t}{|K|} \sum_{\mathscr{E} \in \partial K}\left(|\mathscr{E}| \sum_{q=1}^{Q} \omega_{q} D_{\mathscr{E}, q}^{\operatorname{int}(K)}\right) \\
& =\sum_{\mathscr{E} \in \partial K} \sum_{q=1}^{Q} \varpi_{\mathscr{E}}^{(q)} D_{\mathscr{E}, q}^{\operatorname{int}(K)}+\sum_{q=1}^{\widetilde{Q}} \widetilde{\varpi}_{q} D_{h}^{\operatorname{int}(K)}\left(\widetilde{\mathbf{x}}_{K}^{(q)}\right)-\frac{a \Delta t}{|K|} \sum_{\mathscr{E} \in \partial K}\left(|\mathscr{E}| \sum_{q=1}^{Q} \omega_{q} D_{\mathscr{E}, q}^{\operatorname{int}(K)}\right) \\
& \geq \sum_{\mathscr{E} \in \partial K} \sum_{q=1}^{Q}\left[\omega_{q} D_{\mathscr{E}, q}^{\operatorname{int}(K)}\left(\frac{\varpi_{\mathscr{E}}^{(q)}}{\omega_{q}}-a \Delta t \frac{|\mathscr{E}|}{|K|}\right)\right] \geq 0,
\end{aligned}
$$

where we have used in the above equality the exactness of the quadrature rule (34) for polynomials of degree up to $k$, and in the last inequality the condition (36).

We then prove that $\overline{\mathbf{U}}_{K}^{\Delta t} \cdot \boldsymbol{\xi}^{*}+p_{m}^{*}>0$ for any auxiliary variables $\mathbf{B}^{*} \in \mathbb{R}^{3}$ and $\mathbf{v}^{*} \in \mathbb{B}_{1}(\mathbf{0})$. It follows from (38) that

$$
\overline{\mathbf{U}}_{K}^{\Delta t} \cdot \boldsymbol{\xi}^{*}+p_{m}^{*}=I_{1}+I_{2}+I_{3}+I_{4},
$$

with $I_{1}:=\boldsymbol{\Xi}_{1} \cdot \boldsymbol{\xi}^{*}+\eta, I_{2}:=\boldsymbol{\Xi}_{1} \cdot \boldsymbol{\xi}^{*}+\eta, I_{3}:=\boldsymbol{\Xi}_{3} \cdot \boldsymbol{\xi}^{*}+p_{m}^{*}-2 \eta, I_{4}:=\boldsymbol{\Xi}_{4} \cdot \boldsymbol{\xi}^{*}$, and $\eta:=\frac{a \Delta t}{2|K|} \sum_{\mathscr{E} \in \partial K}|\mathscr{E}| p_{m}^{*}$. We now estimate the lower bounds of $I_{i}$ for $1 \leq i \leq 4$ respectively. Using Lemma 3.7, we deduce that

$$
I_{1}=\frac{a \Delta t}{2|K|} \sum_{\mathscr{E} \in \partial K}\left\{|\mathscr{E}| \sum_{q=1}^{Q} \omega_{q}\left[\left(\mathbf{U}_{\mathscr{E}, q}^{\operatorname{int}(K)}-\frac{1}{a}\left\langle\mathbf{n}_{\mathscr{E}, K}, \mathbf{F}\left(\mathbf{U}_{\mathscr{E}, q}^{\mathrm{int}(K)}\right)\right\rangle\right) \cdot \boldsymbol{\xi}^{*}+p_{m}^{*}\right]\right\}
$$




$$
\begin{aligned}
& \geq \frac{a \Delta t}{2|K|} \sum_{\mathscr{E} \in \partial K}\left\{|\mathscr{E}| \sum_{q=1}^{Q} \omega_{q}\left[\frac{1}{a}\left(\left\langle\mathbf{n}_{\mathscr{E}, K}, \mathbf{v}^{*}\right\rangle p_{m}^{*}-\left\langle\mathbf{n}_{\mathscr{E}, K}, \mathbf{B}_{\mathscr{E}, q}^{\operatorname{int}(K)}\right\rangle\left(\mathbf{v}^{*} \cdot \mathbf{B}^{*}\right)\right)\right]\right\} \\
& =\frac{\Delta t}{2|K|} \sum_{\mathscr{E} \in \partial K}\left\{|\mathscr{E}| \sum_{q=1}^{Q} \omega_{q}\left(-\left\langle\mathbf{n}_{\mathscr{E}, K}, \mathbf{B}_{\mathscr{E}, q}^{\operatorname{int}(K)}\right\rangle\left(\mathbf{v}^{*} \cdot \mathbf{B}^{*}\right)\right)\right\} \\
& =-\frac{\Delta t\left(\mathbf{v}^{*} \cdot \mathbf{B}^{*}\right)}{2|K|} \sum_{\mathscr{E} \in \partial K} \int_{\mathscr{E}}\left\langle\mathbf{n}_{\mathscr{E}, K}, \mathbf{B}_{h}^{\operatorname{int}(K)}\right\rangle d s=:-\frac{\Delta t\left(\mathbf{v}^{*} \cdot \mathbf{B}^{*}\right)}{2|K|} \operatorname{div}_{K}^{\operatorname{int}} \mathbf{B}_{h},
\end{aligned}
$$

where we have used the exactness of the $Q$-point quadrature rule on each interface for polynomials of degree up to $k$. Similarly, we obtain

$$
I_{2} \geq-\frac{\Delta t\left(\mathbf{v}^{*} \cdot \mathbf{B}^{*}\right)}{2|K|} \sum_{\mathscr{E} \in \partial K} \int_{\mathscr{E}}\left\langle\mathbf{n}_{\mathscr{E}, K}, \mathbf{B}_{h}^{\mathrm{ext}(K)}\right\rangle d s=:-\frac{\Delta t\left(\mathbf{v}^{*} \cdot \mathbf{B}^{*}\right)}{2|K|} \operatorname{div}_{K}^{\mathrm{ext}} \mathbf{B}_{h} .
$$

Note $I_{3}=\overline{\mathbf{U}}_{K} \cdot \boldsymbol{\xi}^{*}+p_{m}^{*}-\frac{a \Delta t}{|K|} \sum_{\mathscr{E} \in \partial K}\left(|\mathscr{E}| \sum_{q=1}^{Q} \omega_{q}\left(\mathbf{U}_{\mathscr{E}, q}^{\mathrm{int}(K)} \cdot \boldsymbol{\xi}^{*}+p_{m}^{*}\right)\right)$. Based on the exactness of the quadrature rule (34) for polynomials of degree up to $k$, one has

$$
\begin{aligned}
\overline{\mathbf{U}}_{K} \cdot \boldsymbol{\xi}^{*}+p_{m}^{*} & =\sum_{\mathscr{E} \in \partial K} \sum_{q=1}^{Q} \varpi_{\mathscr{E}}^{(q)}\left(\mathbf{U}_{\mathscr{E}, q}^{\operatorname{int}(K)} \cdot \boldsymbol{\xi}^{*}+p_{m}^{*}\right)+\sum_{q=1}^{\widetilde{Q}} \widetilde{\varpi}_{q}\left(\mathbf{U}_{h}^{\operatorname{int}(K)}\left(\widetilde{\mathbf{x}}_{K}^{(q)}\right) \cdot \boldsymbol{\xi}^{*}+p_{m}^{*}\right) \\
& \geq \sum_{\mathscr{E} \in \partial K} \sum_{q=1}^{Q} \varpi_{\mathscr{E}}^{(q)}\left(\mathbf{U}_{\mathscr{E}, q}^{\operatorname{int}(K)} \cdot \boldsymbol{\xi}^{*}+p_{m}^{*}\right),
\end{aligned}
$$

where $\mathbf{U}_{h}^{\operatorname{int}(K)}\left(\widetilde{\mathbf{x}}_{K}^{(q)}\right) \in \mathcal{G}=\mathcal{G}_{2}$ has been used. It follows that

$$
I_{3} \geq \sum_{\mathscr{E} \in \partial K} \sum_{q=1}^{Q}\left(\mathbf{U}_{\mathscr{E}, q}^{\mathrm{int}(K)} \cdot \boldsymbol{\xi}^{*}+p_{m}^{*}\right)\left(\varpi_{\mathscr{E}}^{(q)}-a \Delta t \omega_{q} \frac{|\mathscr{E}|}{|K|}\right)
$$

Thanks to the inequality (17) constructed in Lemma 3.5, we have

$$
b\left(\mathbf{S}(\mathbf{U}) \cdot \boldsymbol{\xi}^{*}\right) \geq-b\left(\mathbf{v}^{*} \cdot \mathbf{B}^{*}\right)-\frac{|b|}{\sqrt{\rho H}}\left(\mathbf{U} \cdot \boldsymbol{\xi}^{*}+p_{m}^{*}\right), \quad \forall b \in \mathbb{R}, \quad \forall \mathbf{U} \in \mathcal{G} .
$$

It follows that

$$
\begin{array}{r}
\left\langle\mathbf{n}_{\mathscr{E}, K}, \mathbf{B}_{\mathscr{E}, q}^{\operatorname{int}(K)}-\mathbf{B}_{\mathscr{E}, q}^{\operatorname{ext}(K)}\right\rangle \mathbf{S}\left(\mathbf{U}_{\mathscr{E}, q}^{\operatorname{int}(K)}\right) \cdot \boldsymbol{\xi}^{*} \geq\left\langle\mathbf{n}_{\mathscr{E}, K}, \mathbf{B}_{\mathscr{E}, q}^{\operatorname{ext}(K)}-\mathbf{B}_{\mathscr{E}, q}^{\operatorname{int}(K)}\right\rangle\left(\mathbf{v}^{*} \cdot \mathbf{B}^{*}\right) \\
-\left(\rho_{\mathscr{E}, q}^{\operatorname{int}(K)} H_{\mathscr{E}, q}^{\operatorname{int}(K)}\right)^{-\frac{1}{2}}\left|\left\langle\mathbf{n}_{\mathscr{E}, K}, \mathbf{B}_{\mathscr{E}, q}^{\operatorname{int}(K)}-\mathbf{B}_{\mathscr{E}, q}^{\operatorname{ext}(K)}\right\rangle\right|\left(\mathbf{U}_{\mathscr{E}, q}^{\operatorname{int}(K)} \cdot \boldsymbol{\xi}^{*}+p_{m}^{*}\right)
\end{array}
$$

Let $I_{5}:=-\frac{\Delta t}{|K|} \sum_{\mathscr{E} \in \partial K} \sum_{q=1}^{Q} \omega_{q} \sigma_{K, \mathscr{E}, q}\left(\mathbf{U}_{\mathscr{E}, q}^{\mathrm{int}(K)} \cdot \boldsymbol{\xi}^{*}+p_{m}^{*}\right)$. We then obtain a lower bound for $I_{4}$ :

$$
\begin{aligned}
I_{4} & \geq \frac{\Delta t}{2|K|} \sum_{\mathscr{E} \in \partial K}\left[|\mathscr{E}| \sum_{q=1}^{Q} \omega_{q}\left\langle\mathbf{n}_{\mathscr{E}, K}, \mathbf{B}_{\mathscr{E}, q}^{\operatorname{ext}(K)}-\mathbf{B}_{\mathscr{E}, q}^{\operatorname{int}(K)}\right\rangle\left(\mathbf{v}^{*} \cdot \mathbf{B}^{*}\right)\right]+I_{5} \\
& =\frac{\Delta t\left(\mathbf{v}^{*} \cdot \mathbf{B}^{*}\right)}{2|K|}\left(\operatorname{div}_{K}^{\mathrm{ext}} \mathbf{B}_{h}-\operatorname{div}_{K}^{\mathrm{int}} \mathbf{B}_{h}\right)+I_{5} .
\end{aligned}
$$

Thanks to the locally divergence-free property of $\mathbf{B}_{h}(\mathbf{x})$, we have

$$
\operatorname{div}_{K}^{\operatorname{int}} \mathbf{B}_{h}=\sum_{\mathscr{E} \in \partial K} \int_{\mathscr{E}}\left\langle\mathbf{n}_{\mathscr{E}, K}, \mathbf{B}_{h}^{\operatorname{int}(K)}\right\rangle d s=\int_{K} \nabla \cdot \mathbf{B}_{h}^{\operatorname{int}(K)}(\mathbf{x}) d \mathbf{x}=0
$$


where Green's theorem has been used. Combining the estimates (40)-(43) and using (44) and (39), we obtain

$$
\begin{gathered}
\overline{\mathbf{U}}_{K}^{\Delta t} \cdot \boldsymbol{\xi}^{*}+p_{m}^{*} \geq \sum_{\mathscr{E} \in \partial K} \sum_{q=1}^{Q}\left(\mathbf{U}_{\mathscr{E}, q}^{\operatorname{int}(K)} \cdot \boldsymbol{\xi}^{*}+p_{m}^{*}\right)\left(\varpi_{\mathscr{E}}^{(q)}-a \Delta t \omega_{q} \frac{|\mathscr{E}|}{|K|}\right)+I_{5} \\
=\sum_{\mathscr{E} \in \partial K} \sum_{q=1}^{Q} \omega_{q}\left(\mathbf{U}_{\mathscr{E}, q}^{\operatorname{int}(K)} \cdot \boldsymbol{\xi}^{*}+p_{m}^{*}\right)\left[\frac{\varpi_{\mathscr{E}}^{(q)}}{\omega_{q}}-\Delta t \frac{|\mathscr{E}|}{|K|}\left(a+\sigma_{K, \mathscr{E}, q}\right)\right]>0,
\end{gathered}
$$

where the condition (36) has been used in the last inequality. Therefore, we have

$$
\overline{\mathbf{U}}_{K}^{\Delta t} \cdot \boldsymbol{\xi}^{*}+p_{m}^{*}>0, \quad \forall \mathbf{B}^{*} \in \mathbb{R}^{3}, \forall \mathbf{v}^{*} \in \mathbb{B}_{1}(\mathbf{0}),
$$

which, along with $\bar{D}_{K}^{\Delta t}>0$, yield $\overline{\mathbf{U}}_{K}^{\Delta t} \in \mathcal{G}_{2}=\mathcal{G}$. The proof is complete.

Remark 4.5. The quantities $\operatorname{div}_{K}^{\text {int }} \mathbf{B}_{h}$ and $\operatorname{div}_{K}^{\text {ext }} \mathbf{B}_{h}$, defined in the lower bounds in (40) and (41) respectively, denote discrete divergence. They are also defined in [39] to quantify the influence of divergence error on the PCP property of the standard DG schemes, for which the discrete divergencefree condition $\operatorname{div}_{K}^{\text {ext }} \mathbf{B}_{h}=\operatorname{div}_{K}^{\text {int }} \mathbf{B}_{h}=0$ is required. However, the present DG schemes are proven to be PCP without requiring such discrete divergence-free condition, thanks to two key ingredients: the locally divergence-free DG element and a suitable discretization of the source term in the symmetrizable RMHD system (9) which gives $\mathcal{J}_{K}^{(2)}\left(\mathbf{U}_{h}, \mathbf{u}\right)$ in (31). The former leads to zero divergence within each cell, so that the term $\operatorname{div}_{K}^{\text {int }} \mathbf{B}_{h}$ vanishes. The latter brings some new divergence terms, as shown in the lower bound in (43), which exactly offset the divergence term in (41). In other words, these key ingredients help eliminate the effect of divergence error on the PCP property. This feature is similar to the continuous case that the inclusion of source $\mathbf{S}(\mathbf{U}) \nabla \cdot \mathbf{B}$ makes the modified RMHD system (9) able to retain the PCP property even if the magnetic field is not divergence-free. Again, these findings indicate the unity of discrete and continuous objects.

For the first-order DG method $(k=0)$, we have $\left.\mathbf{U}_{h}\right|_{K}(\mathbf{x}) \equiv \overline{\mathbf{U}}_{K}$ and $\mathbb{G}_{h}^{k}=\overline{\mathbb{G}}_{h}^{k}$ so that the PCP and weak PCP properties are equivalent in this case, and the PCP property can be proven under a sharper CFL condition as shown in Theorem 4.6.

ThEOREM 4.6. For the first-order version $(k=0)$ of the semi-discrete DG scheme (31) or (32), the PCP property (25) holds under the following CFL type condition

$$
\Delta t\left(\frac{a}{|K|} \sum_{\mathscr{E} \in \partial K}|\mathscr{E}|+\frac{\left|\operatorname{div}_{K} \mathbf{B}_{h}\right|}{\sqrt{\bar{\rho}_{K} \bar{H}_{K}}}\right)<1, \quad \forall K \in \mathcal{T}_{h},
$$

where $\operatorname{div}_{K} \mathbf{B}_{h}$ denotes a discrete divergence of $\mathbf{B}_{h}$ on the cell $K$ defined by

$$
\operatorname{div}_{K} \mathbf{B}_{h}:=\frac{1}{|K|} \sum_{\mathscr{E} \in \partial K}|\mathscr{E}|\left\langle\mathbf{n}_{\mathscr{E}, K}, \frac{\overline{\mathbf{B}}_{K}+\overline{\mathbf{B}}_{K_{\mathscr{E}}}}{2}\right\rangle
$$

with $K_{\mathscr{E}}$ denoting the adjacent cell that shares the edge $\mathscr{E}$ with the cell $K$. In (45)-(46), the notations $\bar{\rho}_{K}, \bar{H}_{K}$ and $\overline{\mathbf{B}}_{K}$ denote the rest-mass density, specific enthalpy, and magnetic field corresponding to $\overline{\mathbf{U}}_{K}$, respectively.

Proof. The proof is similar to that of Theorem 4.4 and is thus omitted.

4.3. The PCP limiting operator $\Pi_{h}$. We now present the PCP limiting operator $\Pi_{h}$ : $\overline{\mathbb{G}}_{h}^{k} \longrightarrow \mathbb{G}_{h}^{k}$, which limits the numerical solutions from $\overline{\mathbb{G}}_{h}^{k}$ to $\mathbb{G}_{h}^{k}$ via a simple scaling PCP limiter [39] as extension of the positivity-preserving limiter [47]. For any $\mathbf{U}_{h} \in \overline{\mathbb{G}}_{h}^{k}$, we construct the limited solution $\Pi_{h} \mathbf{U}_{h}=: \widetilde{\mathbf{U}}_{h} \in \mathbb{G}_{h}^{k}$ as follows.

Let $\left.\mathbf{U}_{h}\right|_{K}=: \mathbf{U}_{K}(\mathbf{x})$. Note $\overline{\mathbf{U}}_{K} \in \mathcal{G}=\mathcal{G}_{1}, \forall K \in \mathcal{T}_{h}$. To avoid the effect of the rounding error, we introduce a sufficiently small positive number $\epsilon$ such that $\overline{\mathbf{U}}_{K} \in \mathcal{G}_{\epsilon}$ for all $K \in \mathcal{T}_{h}$, where 
$\mathcal{G}_{\epsilon}=\left\{\mathbf{U}=(D, \mathbf{m}, \mathbf{B}, E)^{\top}: D \geq \epsilon, q(\mathbf{U}) \geq \epsilon, \Psi_{\epsilon}(\mathbf{U}) \geq 0\right\}$ is a convex set [39], with $\Psi_{\epsilon}(\mathbf{U}):=\Psi\left(\mathbf{U}_{\epsilon}\right)$ and $\mathbf{U}_{\epsilon}:=(D, \mathbf{m}, \mathbf{B}, E-\epsilon)^{\top}$. For each $K$, to construct $\widetilde{\mathbf{U}}_{K}(\mathbf{x}):=\left.\widetilde{\mathbf{U}}_{h}\right|_{K}$, we proceed as follows. First, we define $\widehat{\mathbf{U}}_{K}(\mathbf{x}):=\left(\widehat{D}_{K}(\mathbf{x}), \mathbf{m}_{K}(\mathbf{x}), \mathbf{B}_{K}(\mathbf{x}), E_{K}(\mathbf{x})\right)^{\top}$ with $\widehat{D}_{K}(\mathbf{x})=\theta_{1}\left(D_{K}(\mathbf{x})-\bar{D}_{K}\right)+\bar{D}_{K}$, and $\theta_{1}=\min \left\{1,\left(\bar{D}_{K}-\epsilon\right) /\left(\bar{D}_{K}-\min _{\mathbf{x} \in \mathbb{S}_{K}} D_{K}(\mathbf{x})\right)\right\}$. Then, we define $\check{\mathbf{U}}_{K}(\mathbf{x}):=\left(\theta_{2}\left(\widehat{D}_{K}(\mathbf{x})-\bar{D}_{K}\right)+\right.$ $\left.\bar{D}_{K}, \theta_{2}\left(\mathbf{m}_{K}(\mathbf{x})-\overline{\mathbf{m}}_{K}\right)+\overline{\mathbf{m}}_{K}, \mathbf{B}_{K}(\mathbf{x}), \theta_{2}\left(E_{K}(\mathbf{x})-\bar{E}_{K}\right)+\bar{E}_{K}\right)^{\top}$ with $\theta_{2}=\min \left\{1,\left(q\left(\overline{\mathbf{U}}_{K}\right)-\right.\right.$ $\left.\epsilon) /\left(q\left(\overline{\mathbf{U}}_{K}\right)-\min _{\mathbf{x} \in \mathbb{S}_{K}} q\left(\check{\mathbf{U}}_{K}(\mathbf{x})\right)\right)\right\}$. Finally, we define

$$
\widetilde{\mathbf{U}}_{K}(\mathbf{x})=\theta_{3}\left(\check{\mathbf{U}}_{K}(\mathbf{x})-\overline{\mathbf{U}}_{K}\right)+\overline{\mathbf{U}}_{K},
$$

where $\theta_{3}=\min _{\mathbf{x} \in \mathbb{S}_{K}} \tilde{\theta}(\mathbf{x})$. Here $\tilde{\theta}(\mathbf{x})=1$ if $\Psi_{\epsilon}\left(\check{\mathbf{U}}_{K}(\mathbf{x})\right) \geq 0$; otherwise $\tilde{\theta}(\mathbf{x}) \in[0,1)$ solves $\Psi_{\epsilon}((1-$ $\left.\tilde{\theta}) \overline{\mathbf{U}}_{K}+\tilde{\theta} \check{\mathbf{U}}_{K}(\mathbf{x})\right)=0$, which has a unique solution for the unknown $\tilde{\theta} \in[0,1)$.

Lemma 4.7. For any $\mathbf{U}_{h} \in \overline{\mathbb{G}}_{h}^{k}$, one has $\boldsymbol{\Pi}_{h} \mathbf{U}_{h}=\widetilde{\mathbf{U}}_{h} \in \mathbb{G}_{h}^{k}$.

Proof. The above procedure indicates that, for $\forall K \in \mathcal{T}_{h}$, the limited solution defined by (47) satisfies $\widetilde{\mathbf{U}}_{K}(\mathbf{x}) \in \mathcal{G}_{\epsilon} \subset \mathcal{G}_{1}=\mathcal{G}, \forall \mathbf{x} \in \mathbb{S}_{K}$, and $\frac{1}{|K|} \int_{K} \widetilde{\mathbf{U}}_{K} d \mathbf{x}=\overline{\mathbf{U}}_{K}$. Besides, the limited magnetic field $\widetilde{\mathbf{B}}_{K}(\mathbf{x})$ keeps locally divergence-free within $K$.

Remark 4.8. The PCP limiting operator $\boldsymbol{\Pi}_{h}$ keeps the conservativeness (26). Such a limiter does not destroy the high-order accuracy; see [46, 47, 45].

4.4. The PCP property of fully discrete schemes. The PCP property of our fully discrete Runge-Kutta DG scheme (27) is proven in the following theorems.

TheOrem 4.9. Assume that $\mathbf{U}_{h}^{(0)}=\mathbf{U}_{h}^{n} \in \mathbb{G}_{h}^{k}$, then the solutions $\mathbf{U}_{h}^{(i)}, 1 \leq i \leq N_{r}$ computed by the proposed DG scheme (27) belong to $\mathbb{G}_{h}^{k}$, under the CFL condition

$$
\Delta t_{n} \leq \min _{i, \ell} \frac{\varpi_{\mathscr{E}}^{(q)}|K|}{\beta_{i \ell} \omega_{q}\left(a+\sigma_{K, \mathscr{E}, q}\left(\mathbf{U}_{h}^{(i)}\right)\right)|\mathscr{E}|}, \quad 1 \leq q \leq Q, \forall \mathscr{E} \in \partial K, \forall K \in \mathcal{T}_{h} .
$$

Proof. We prove it by the second principle of mathematical induction for $i$. The hypothesis implies $\mathbf{U}_{h}^{(i)} \in \mathbb{G}_{h}^{k}$ for $i=0$. Assume that $\mathbf{U}_{h}^{(\ell)} \in \mathbb{G}_{h}^{k}, 1 \leq \ell \leq i-1$. Thanks to the weak PCP property (25) in Theorem 4.4, we have $\mathbf{U}_{h}^{(\ell)}+\beta_{i \ell} \Delta t_{n} \mathbf{L}_{h}\left(\mathbf{U}_{h}^{(\ell)}\right) \in \overline{\mathbb{G}}_{h}^{k}, 1 \leq \ell \leq i-1$ under the CFL condition (48). The convexity of $\overline{\mathbb{G}}_{h}^{k}$ in Lemma 4.2 implies $\sum_{\ell=0}^{i-1}\left[\alpha_{i \ell}\left(\mathbf{U}_{h}^{(\ell)}+\beta_{i \ell} \Delta t_{n} \mathbf{L}_{h}\left(\mathbf{U}_{h}^{(\ell)}\right)\right)\right] \in \overline{\mathbb{G}}_{h}^{k}$. Since the PCP limiting operator $\boldsymbol{\Pi}_{h}$ maps the numerical solutions from $\overline{\mathbb{G}}_{h}^{k}$ to $\mathbb{G}_{h}^{k}$, we obtain $\mathbf{U}_{h}^{(i)} \in \mathbb{G}_{h}^{k}$ by (27). Using the principle of induction, we have $\mathbf{U}_{h}^{(i)} \in \mathbb{G}_{h}^{k}$ for all $i \in\left\{0,1, \ldots, N_{r}\right\}$.

TheOREM 4.10. Under the CFL condition (48), the proposed fully discrete Runge-Kutta DG scheme (27) always preserves $\mathbf{U}_{h}^{n} \in \mathbb{G}_{h}^{k}$ for all $n \in \mathbb{N}$.

Proof. Since $\mathbf{P}_{w}(\mathbf{U}(\mathbf{x}, 0)) \in \overline{\mathbb{G}}_{h}^{k}$ as indicated by Lemma 4.2, we known $\mathbf{U}_{h}^{0} \in \mathbb{G}_{h}^{k}$. With the help of Theorem 4.9, we obtain the conclusion by induction for $n$.

4.5. Illustration of some details on Cartesian meshes. Assume that the mesh is rectangular with cells $\left\{\left[x_{i-1 / 2}, x_{i+1 / 2}\right] \times\left[y_{\ell-1 / 2}, y_{\ell+1 / 2}\right]\right\}$ and spatial step-sizes $\Delta x_{i}=x_{i+1 / 2}-x_{i-1 / 2}$ and $\Delta y_{\ell}=y_{\ell+1 / 2}-y_{\ell-1 / 2}$ in $x$ - and $y$-directions respectively. Let $\mathbb{S}_{i}^{x}=\left\{x_{i}^{(q)}\right\}_{q=1}^{Q}$ and $\mathbb{S}_{\ell}^{y}=\left\{y_{\ell}^{(q)}\right\}_{q=1}^{Q}$ denote the $Q$-point Gauss quadrature nodes in the intervals $\left[x_{i-1 / 2}, x_{i+1 / 2}\right]$ and $\left[y_{\ell-1 / 2}, y_{\ell+1 / 2}\right]$ respectively. For the cell $K=\left[x_{i-1 / 2}, x_{i+1 / 2}\right] \times\left[y_{\ell-1 / 2}, y_{\ell+1 / 2}\right]$, the point set $\breve{\mathbb{S}}_{K}$ required in (35) is $\mathbb{S}_{i}^{x} \otimes \mathbb{S}_{\ell}^{y}$, and the set $\widehat{\mathbb{S}}_{K}$ is given by (cf. [46])

$$
\widehat{\mathbb{S}}_{K}=\left(\widehat{\mathbb{S}}_{i}^{x} \otimes \mathbb{S}_{\ell}^{y}\right) \cup\left(\mathbb{S}_{i}^{x} \otimes \widehat{\mathbb{S}}_{\ell}^{y}\right),
$$


where $\widehat{\mathbb{S}}_{i}^{x}=\left\{\widehat{x}_{i}^{(\mu)}\right\}_{\mu=1}^{\mathrm{L}}$ and $\widehat{\mathbb{S}}_{\ell}^{y}=\left\{\widehat{y}_{\ell}^{(\mu)}\right\}_{\mu=1}^{\mathrm{L}}$ denote the L-point (L $\left.\geq \frac{k+3}{2}\right)$ Gauss-Lobatto quadrature nodes in the intervals $\left[x_{i-1 / 2}, x_{i+1 / 2}\right]$ and $\left[y_{\ell-1 / 2}, y_{\ell+1 / 2}\right]$ respectively. With $\widehat{\mathbb{S}}_{K}$ in (49), a special quadrature [46] satisfying (34) can be constructed:

$$
\begin{aligned}
\frac{1}{|K|} \int_{K} u(\mathbf{x}) d \mathbf{x} & =\frac{\Delta x_{i} \widehat{\omega}_{1}}{\Delta x_{i}+\Delta y_{\ell}} \sum_{q=1}^{Q} \omega_{q}\left(u\left(x_{i}^{(q)}, y_{\ell-\frac{1}{2}}\right)+u\left(x_{i}^{(q)}, y_{\ell+\frac{1}{2}}\right)\right) \\
& +\frac{\Delta y_{\ell} \widehat{\omega}_{1}}{\Delta x_{i}+\Delta y_{\ell}} \sum_{q=1}^{Q} \omega_{q}\left(u\left(x_{i-\frac{1}{2}}, y_{\ell}^{(q)}\right)+u\left(x_{i+\frac{1}{2}}, y_{\ell}^{(q)}\right)\right) \\
& +\frac{\Delta x_{i}}{\Delta x_{i}+\Delta y_{\ell}} \sum_{\mu=2}^{\mathrm{L}-1} \sum_{q=1}^{Q} \widehat{\omega}_{\mu} \omega_{q} u\left(x_{i}^{(q)}, \widehat{y}_{\ell}^{(\mu)}\right) \\
& +\frac{\Delta y_{\ell}}{\Delta x_{i}+\Delta y_{\ell}} \sum_{\mu=2}^{\mathrm{L}-1} \sum_{q=1}^{Q} \widehat{\omega}_{\mu} \omega_{q} u\left(\widehat{x}_{i}^{(\mu)}, y_{\ell}^{(q)}\right), \quad \forall u \in \mathbb{P}^{k}(K),
\end{aligned}
$$

where $\left\{\widehat{w}_{\mu}\right\}_{\mu=1}^{\mathrm{L}}$ are the weights of the L-point Gauss-Lobatto quadrature. If labeling the bottom, right, top and left edges of $K$ as $\mathscr{E}_{1}, \mathscr{E}_{2}, \mathscr{E}_{3}$ and $\mathscr{E}_{4}$, respectively, then (50) implies $\varpi_{\mathscr{E}_{j}}^{(q)}=\frac{\Delta x_{i} \widehat{\omega}_{1} \omega_{q}}{\Delta x_{i}+\Delta y_{\ell}}, j=$ 1,$3 ; \varpi_{\mathscr{E}_{j}}^{(q)}=\frac{\Delta y_{\ell} \widehat{\iota}_{1} \omega_{q}}{\Delta x_{i}+\Delta y_{\ell}}, j=2,4$. According to Theorem 4.9, the CFL condition (48) for our PCP schemes on Cartesian meshes is

$$
\Delta t_{n}\left(\frac{1}{\Delta x_{i}}+\frac{1}{\Delta y_{\ell}}\right)<\min _{m, s, q} \frac{\widehat{\omega}_{1}}{\beta_{m s}\left(a+\sigma_{K, \mathscr{E}_{j}, q}\left(\mathbf{U}_{h}^{(m)}\right)\right)}, \quad \forall K \in \mathcal{T}_{h}, 1 \leq j \leq 4
$$

where $\widehat{\omega}_{1}=\frac{1}{\mathrm{~L}(\mathrm{~L}-1)}$. Since $\sigma_{K, \mathscr{E}_{j}, q}\left(\mathbf{U}_{h}^{(m)}\right)$ depends on the numerical solutions at intermediate RungeKutta stages, it is difficult to rigorously enforce the condition (51). Note that $\sigma_{K, \mathscr{E}_{j}, q}\left(\mathbf{U}_{h}^{(m)}\right)$ is proportional to the jump of the normal magnetic component across cell interface, which is zero for the exact solution. Thus $\sigma_{K, \mathscr{E}_{j}, q}$ is small and at the level of truncation error. Thus we suggest $\Delta t_{n}=\frac{C_{\mathrm{cfl}}}{a \max _{m, s} \beta_{m s}}\left(\frac{1}{\Delta x_{i}}+\frac{1}{\Delta y_{\ell}}\right)^{-1}$, with the CFL number $C_{\mathrm{cfl}}$ (slightly) smaller than $\widehat{\omega}_{1}$, which works robustly in our numerical tests. For the third-order SSP Runge-Kutta method (28), $\max _{m, s} \beta_{m s}=1$.

5. Numerical tests. This section conducts numerical tests on several $2 \mathrm{D}$ challenging RMHD problems with either strong discontinuities, low plasma-beta $\beta:=p / p_{m}$, or low rest-mass density or pressure, to demonstrate our theoretical analysis, as well as the accuracy, high-resolution and robustness of the proposed PCP methods. Without loss of generality, we focus on the proposed PCP third-order $(k=2)$ DG methods on uniform Cartesian meshes, with the third-order SSP RungeKutta time discretization (27)-(28). Unless otherwise stated, all the computations are restricted to the ideal EOS (3) with $\gamma=5 / 3$, and the CFL number is set as 0.15 .

5.1. Smooth problems. Two smooth problems are tested to check the accuracy of our method. The first one is similar to those simulated in [38, 24], and its exact solution is $(\rho, \mathbf{v}, \mathbf{B}, p)(x, y, t)=$ $\left(1+0.9999999 \sin (2 \pi(x+y-1.1 t)), 0.9,0.2,0,1,1,1,10^{-2}\right)$, which describes a RMHD sine wave (with very low density and low pressure) fast propagating in the domain $\Omega=[0,1]^{2}$ with a large velocity $|\mathbf{v}| \approx 0.922 c$. The second problem describes Alfvén waves propagating periodically in $\Omega=$ $[0, \sqrt{2}]^{2}$ with a speed of $0.9 c$ higher than that in [49]. The exact solution of this problem is given by $\rho(x, y, t)=1, v_{1}(x, y, t)=-0.9 \sin (2 \pi(\varsigma+t / \kappa)) \sin \alpha, v_{2}(x, y, t)=0.9 \sin (2 \pi(\varsigma+t / \kappa)) \cos \alpha$, $v_{3}(x, y, t)=0.9 \cos (2 \pi(\varsigma+t / \kappa)), B_{1}(x, y, t)=\cos \alpha+\kappa v_{1}(x, y, t), B_{2}(x, y, t)=\sin \alpha+\kappa v_{2}(x, y, t)$, $B_{3}(x, y, t)=\kappa v_{3}(x, y, t), p(x, y, t)=0.1$, where $\kappa=\sqrt{1+\rho H W^{2}}$ and $\varsigma=x \cos \alpha+y \sin \alpha$ with $\alpha=\pi / 4$.

In the computations, the domain $\Omega$ is divided into $N \times N$ uniform rectangular cells with $N \in$ $\{10,20,40,80,160,320,640\}$, and periodic boundary conditions are used. Fig. 1 shows the numerical errors at $t=1$ in the numerical solutions computed by the PCP third-order DG method at different 
grid resolutions. It is seen that the magnitudes of the errors are reduced as we refine the mesh. Moreover, the expected third-order convergence rate is observed, indicating that our discretization of the added source term in the symmetrizable RMHD system (9) and the PCP limiting procedure both maintain the desired accuracy, as expected.
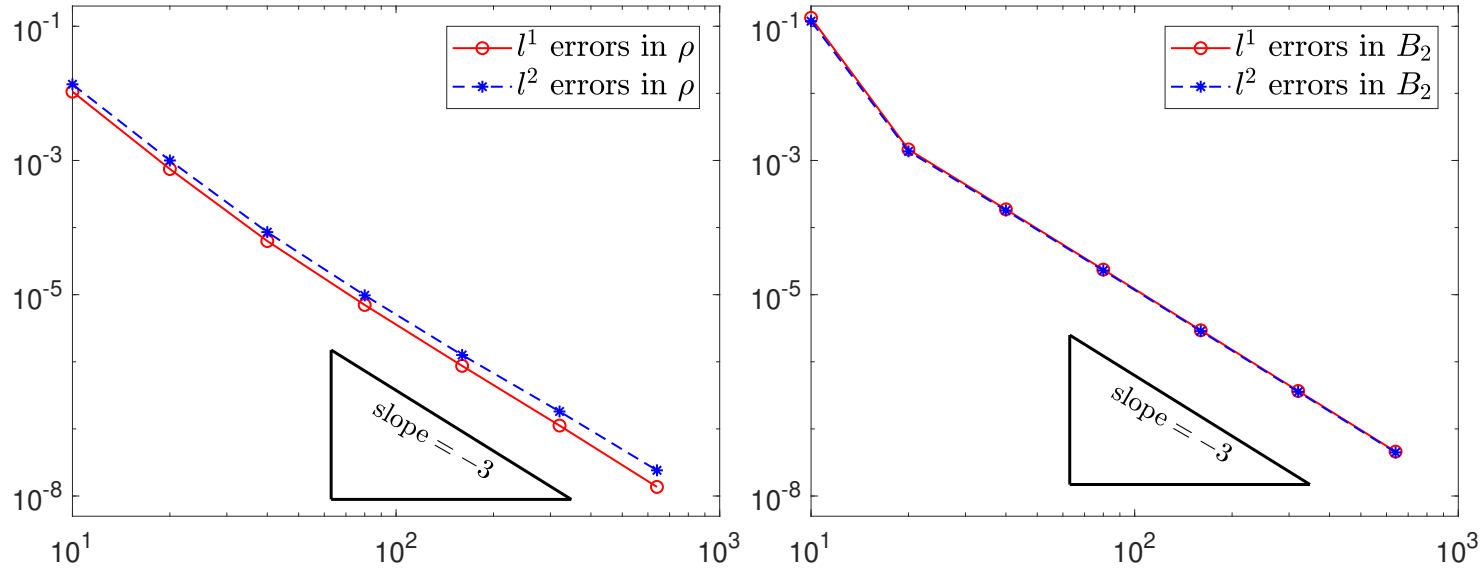

Fig. 1: Numerical errors in $l^{1}$ and $l^{2}$ norms at $t=1$ for the first smooth problem (left) and the second smooth problem (right). The horizontal axis represents the value of $N$.

To verify the capability of the proposed PCP methods in resolving complicated wave configurations, we will simulate an Orszag-Tang problem, three blast problems and two astrophysical jets. For these problems, before the PCP limiting procedure, the WENO limiter [25] with locally divergencefree WENO reconstruction [49] is implemented with the local characteristic decomposition to enhance the numerical stability of high-oder DG methods in resolving the strong discontinuities. The WENO limiter is only used in the "trouble" cells adaptively detected by the indicator in [16].

5.2. Orszag-Tang problem. This test simulates an Orszag-Tang problem for the RMHD [31]. Initially, the domain $\Omega=[0,2 \pi]^{2}$ is filled with relativistically hot gas, and periodic boundary conditions are used. We set the adiabatic index $\Gamma=4 / 3$, the initial rest-mass density $\rho=1$ and thermal pressure $p=10$. The initial velocity field of the fluid is $\mathbf{v}(x, y, 0)=(-A \sin (y), A \sin (x), 0)$, where the parameter $A=0.99 / \sqrt{2}$ so that the maximum velocity is $0.99 c$ (corresponding Lorentz factor $\approx 7.09$ ). The magnetic field is initialized at $\mathbf{B}(x, y, 0)=(-\sin y, \sin (2 x), 0)$. Although the initial solution is smooth, complicated wave structures are formed as time increases, and turbulence behavior will be produced eventually. Fig. 2 gives the numerical results computed by the third-order PCP method on $600 \times 600$ uniform grids. One can see that the complicated flow structures are well captured by our method with high resolution and agree with those presented in $[31,36]$. In this test, we observe that it is necessary to enforce the DG solution in $\mathbb{G}_{n}^{k}$ by the PCP limiting procedure, otherwise the code would break down at time $t \approx 1.98$.

5.3. Blast problems. Blast problem is a benchmark test for RMHD numerical schemes. Simulating a RMHD blast problem with strong magnetic field is difficult, because nonphysical quantities, e.g., negative pressure, are very likely to be produced in the numerical simulation. Our setup is similar to those in $[21,7,1,44]$. Initially, the domain $\Omega=[-6,6]^{2}$ is filled with a homogeneous gas at rest with adiabatic index $\Gamma=4 / 3$. The explosion zone $(r<0.8)$ has a density of $10^{-2}$ and a pressure of 1 , while the ambient medium $(r>1)$ has a density of $10^{-4}$ and a pressure of $p_{a}=5 \times 10^{-4}$, where $r=\sqrt{x^{2}+y^{2}}$. A linear taper is applied to the density and pressure for $r \in[0.8,1]$. The magnetic field is initialized in the $x$-direction as $B_{a}$. As $B_{a}$ is set larger, the initial ambient magnetization becomes higher $\left(\beta_{a}:=p_{a} / p_{m}\right.$ becomes lower) and this test becomes more challenging. In the literature $[21,7,1], B_{a}$ is usually specified as 0.1 , which corresponds to a moderate magnetized case $\left(\beta_{a}=0.1\right)$. A more strongly magnetized case with $B_{a}=0.5$ was tested in [44], corresponding to a lower plasma-beta $\beta_{a}=4 \times 10^{-3}$. Many existing methods in the literature require some artificial treatments for the strongly magnetized case; see e.g., [15, 21, 7]. It was reported in [7] that the 

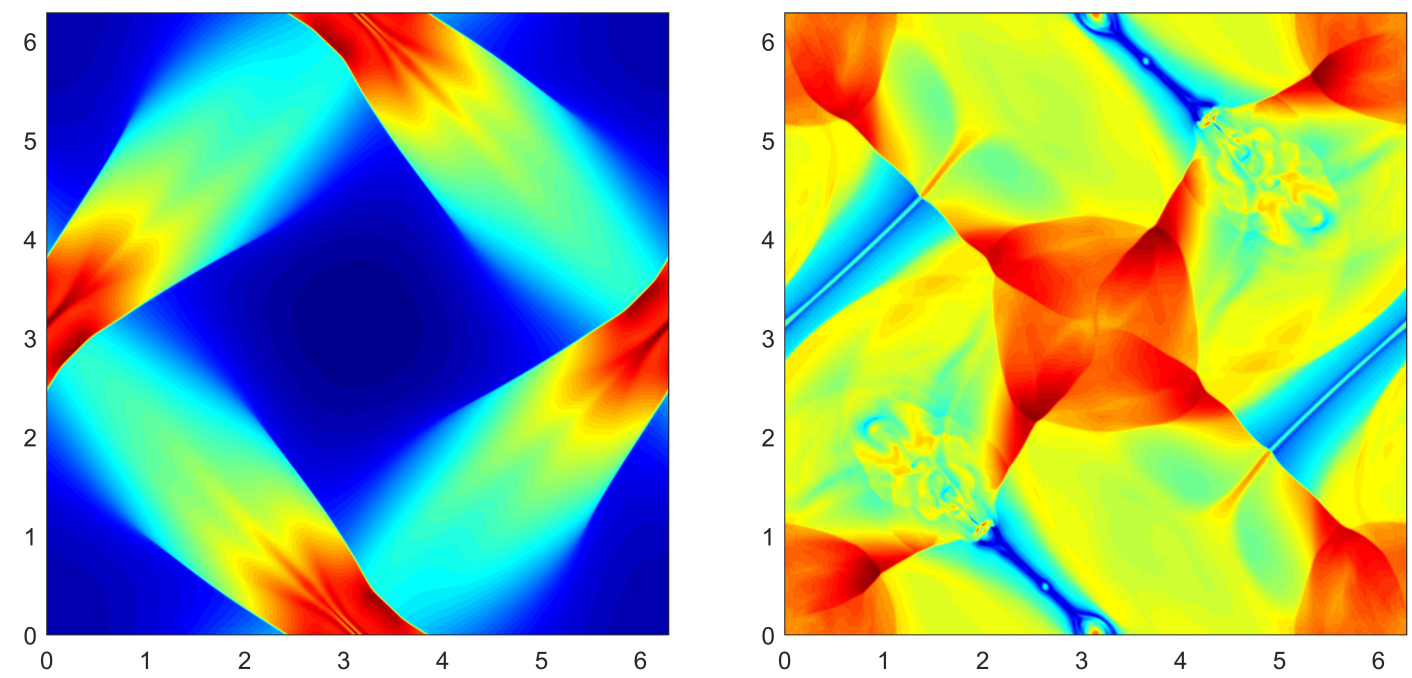

Fig. 2: Schlieren images of $\log (\rho)$ at $t=2.818127$ (left) and $t=6.8558$ (right) for the Orszag-Tang problem.

RMHD code ECHO was not able to run this test with $B_{a}>0.1$ if no ad hoc numerical strategy was employed.

In order to examine the robustness and PCP property of our method, we run this test with different $B_{a} \in\{0.1,0.5,20,100,2000\}$. These five configurations include the two in $[21,7,1,44]$ and three much more challenging cases. We observe that our PCP methods are able to successfully simulate all these test cases without any artificial treatments. Fig. 3 shows our numerical results at $t=4$, obtained using our third-order PCP method on $400 \times 400$ mesh grids, for three configurations: a moderate magnetized case $\left(B_{a}=0.1\right)$, a relatively strongly magnetized case $\left(B_{a}=0.5\right)$, and a extremely strongly magnetized case $\left(B_{a}=2000\right)$. For the first two cases, our results agree quite well with those reported in [44,1,39]. The wave patterns for $B_{a}=20$ and $B_{a}=100$ are similar to that for $B_{a}=2000$ and thus omitted here. From Fig. 3, we see that the wave pattern for $B_{a}=0.1$ is composed by two main waves, an external fast and a reverse shock waves. The former is almost circular, while the latter is elliptic. The magnetic field is essentially confined between them, while the inner region is almost devoid of magnetization. In the case of $B_{a}=0.5$, the external circular fast shock is clearly visible but very weak. For $B_{a} \geq 20$, the external circular fast shock becomes much weaker and is only visible in the magnetic field.

As far as we know, successful simulations of an extreme RMHD blast test with $B_{a}=2000$ and so low plasma-beta $\left(\beta_{a}=2.5 \times 10^{-10}\right)$ have not been reported in the literature. We also notice that, if the PCP limiter is turned off in the strongly magnetized tests $\left(B_{a} \geq 0.5\right)$, nonphysical numerical solutions exceeding the set $\mathbb{G}_{n}^{k}$ will appear in the simulations, and the DG code will break down. We have also performed the above tests by dropping the discrete symmetrization source term $\mathcal{J}_{K}^{(2)}\left(\mathbf{U}_{h}, \mathbf{u}\right)$ in our PCP scheme (31) and keeping the PCP and WENO limiters turned on. The resulting scheme is actually the locally divergence-free DG method with PCP and WENO limiters for the conservative RMHD system (1). We observed that this scheme, which is generally not PCP in theory [39], cannot run the tests with $B_{a} \in\{100,2000\}$. This demonstrates the importance and necessity of including the proper discretization of the symmetrization source term for the PCP property of the DG schemes.

5.4. Astrophysical jets. The last test is to simulate two relativistic jets, where the internal energy is exceedingly small compared to the kinetic energy so that negative pressure could be easily produced in numerical simulation. Moreover, there may exist strong shock wave, shear flow and interface instabilities in high-speed jet flows. Successful simulation of such jet flows is indeed challenging; cf. $[47,38,24,40,35]$.

We consider a pressure-matched highly supersonic RHD jet model from [40] and add a magnetic field so as to simulate the RMHD jet flows. Initially, the domain $[-12,12] \times[0,25]$ is filled with a static uniform medium with an unit rest-mass density. A RMHD jet of Mach number $M_{b}=50$ is injected 

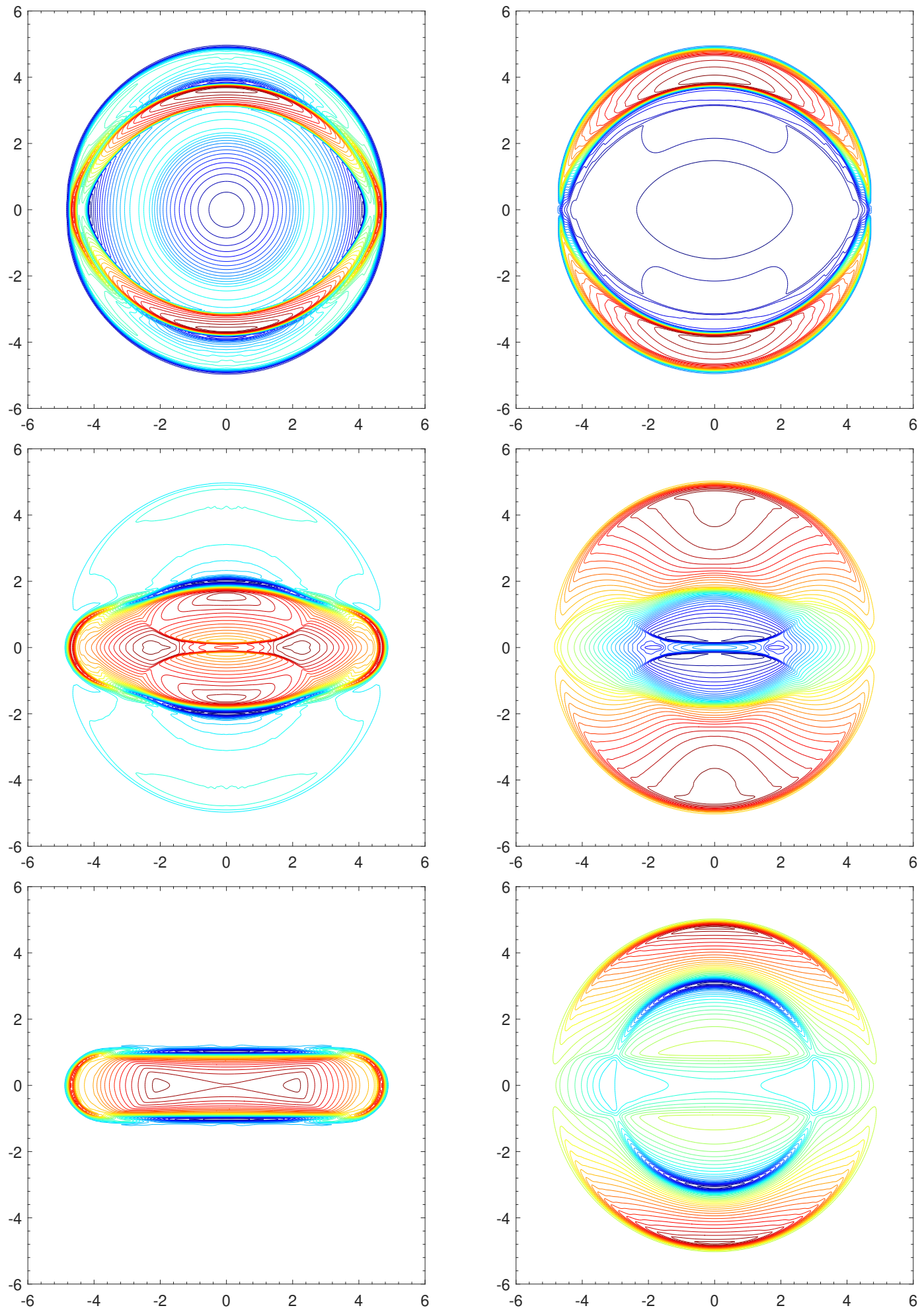

Fig. 3: Contour plots of $\log (\rho)$ (left) and $|\mathbf{B}|$ (right) for the blast problems at $t=4$. Forty equally spaced contour lines are displayed. Top: the moderate magnetized case $\left(B_{a}=0.1, \beta_{a}=0.1\right)$; middle: the relatively strongly magnetized case $\left(B_{a}=0.5, \beta_{a}=4 \times 10^{-3}\right)$; bottom: the extremely strongly magnetized case $\left(B_{a}=2000, \beta_{a}=2.5 \times 10^{-10}\right)$. 
in the $y$-direction through the inlet part $(|x| \leq 0.5)$ on the bottom boundary $(y=0)$ with a density of $\rho_{b}=0.1$, a pressure equal to the ambient pressure, and a speed of $v_{b}=0.99 \mathrm{c}$. The corresponding initial Lorentz factor $W \approx 7.09$ and the relativistic Mach number $M_{r}:=M_{b} W / W_{s} \approx 354.37$, where $W_{s}=1 / \sqrt{1-c_{s}^{2}}$ is the Lorentz factor associated with the local sound speed $c_{s}$. The exceedingly high Mach number and large Lorentz factor render the simulation of this problem very challenging. The fixed inflow condition is specified on the nozzle $\{y=0,|x| \leq 0.5\}$, while the other boundary conditions are outflow. A magnetic field with a magnitude of $B_{a}$ is initialized along the $y$-direction. The presence of magnetic field makes this test more extreme. We simulate a non-magnetized case with $B_{a}=0$ and a strongly magnetized case with $B_{a}=\sqrt{2000 p}$ (the corresponding plasma-beta $\left.\beta_{a}=10^{-3}\right)$. The computational domain is taken as $[0,12] \times[0,30]$ and divided into $240 \times 500$ uniform cells with the reflecting boundary condition on $\{x=0,0 \leq y \leq 25\}$.
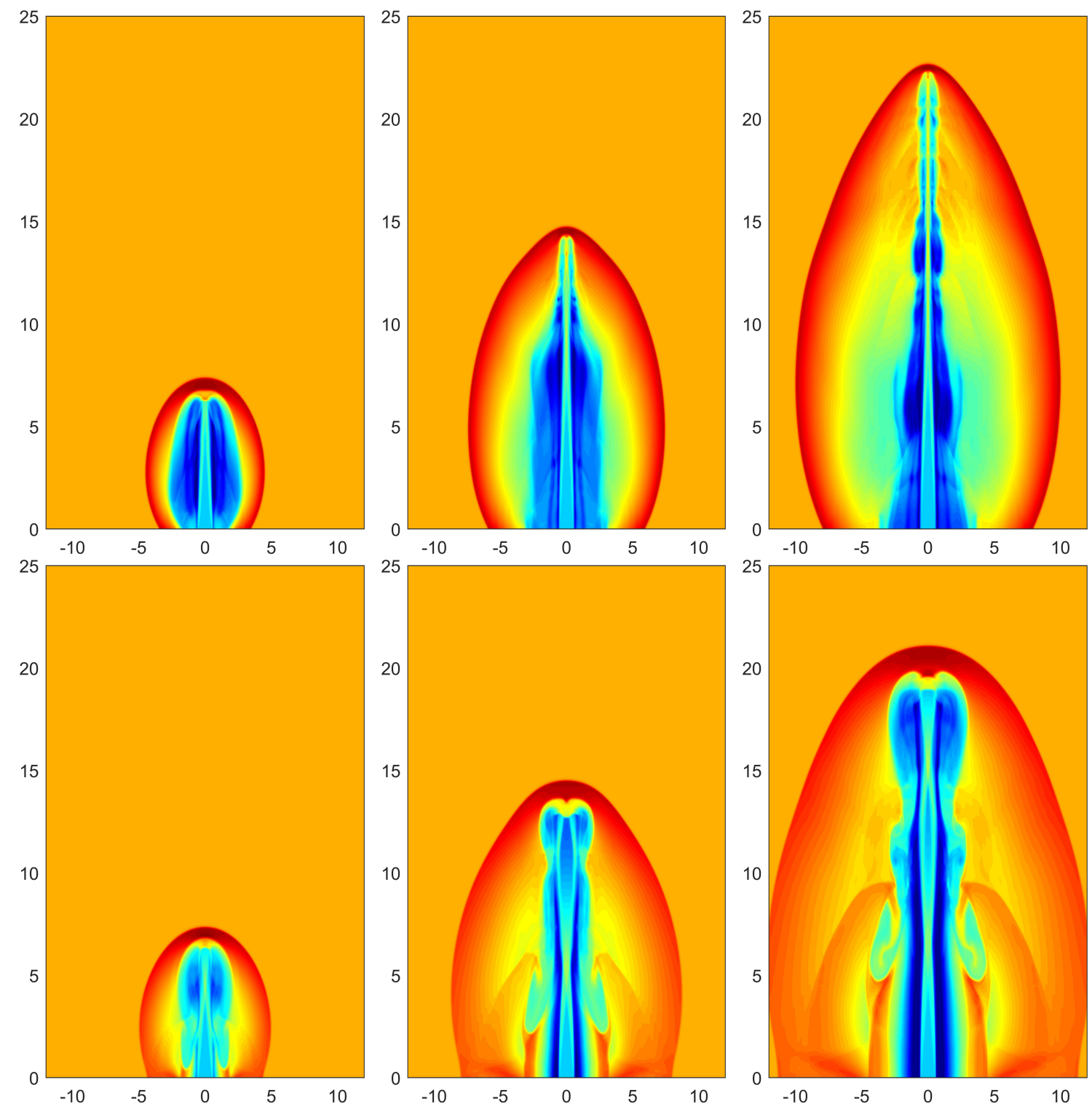

Fig. 4: Schlieren images of $\log (\rho)$ for the relativistic jets at $t=10,20$, and 30 (from left to right). Top: the non-magnetized case; bottom: the strongly magnetized case.

Fig. 4 displays the schlieren images of the rest-mass density logarithm within the domain $[-12,12] \times$ $[0,25]$ at $t=10,20$ and 30, computed by using the proposed third-order PCP DG method, for the 
non-magnetized case and the strongly magnetized case, respectively. Those plots clearly show the dynamics of the relativistic jets. We observe that the Mach shock wave at the jet head and the beam/cocoon interface are well captured during the whole simulation. The wave patterns for the non-magnetized case are in good agreement with those computed in [40]. Due to the effect of the strong magnetic field, the flow structures of the strongly magnetized case are quite different from those of the non-magnetized case. Our PCP method exhibits good robustness in such extreme tests. It is observed that if the PCP limiter is turned off, the simulation will break down after several time steps due to nonphysical numerical solutions. In addition, if dropping the discrete symmetrization source term $\mathcal{J}_{K}^{(2)}\left(\mathbf{U}_{h}, \mathbf{u}\right)$ in our PCP scheme (31), we find the cell averages of the DG solutions will exceed the set $\mathcal{G}_{1}$ and the scheme will fail in the strongly magnetized test. Again, this demonstrates the importance of including the proper discretization of the symmetrization source term for the PCP property.

6. Conclusions. We have proposed a framework of constructing provably PCP high-order DG schemes for the multidimensional RMHD with a general EOS on general meshes. The schemes are based on a suitable, locally divergence-free DG discretization of symmetrizable RMHD equations, which have accommodated the PCP property at PDE level. The resulting DG schemes with strongstability-preserving time discretizations satisfy a weak PCP property, which implies that a simple limiter can enforce the PCP property without losing conservation and high order accuracy. Most notably, we rigorously prove the PCP property by using a novel "quasi-linearization" approach to handle the highly nonlinear physical constraints, technical splitting to offset the influence of divergence error, and sophisticated estimates to analyze the beneficial effect of the symmetrization source term. Several demanding numerical examples, including strongly magnetized bast problem with extremely low plasma-beta $\left(2.5 \times 10^{-10}\right)$ and highly supersonic RMHD jets, have been tested and demonstrated the effectiveness and robustness of the proposed PCP schemes. In the context of RMHD, our findings furthered the understanding, at both discrete and continuous levels, of the intrinsic connection between the PCP property and divergence-free condition, indicating the unity of discrete and continuous objects.

Appendix A. Proof of Proposition 2.1. Due to the assumption that the the strong solution of the initial-value problem exists for $\mathbf{x} \in \mathbb{R}^{d}$ and $0 \leq t \leq T$, the Lorentz factor $W$ does not blow up, and then $|\mathbf{v}(\mathbf{x}, t)|<1$ for all $\mathbf{x} \in \mathbb{R}^{d}$ and $0 \leq t \leq T$. Let $\frac{\mathcal{D}}{\mathcal{D} t}:=\frac{\partial}{\partial t}+\mathbf{v}(\mathbf{x}, t) \nabla$. be the directional derivative along the direction

$$
\frac{d \mathbf{x}}{d t}=\mathbf{v}(\mathbf{x}, t)
$$

For any $(\overline{\mathbf{x}}, \bar{t}) \in \mathbb{R}^{d} \times \mathbb{R}_{+}$, let $\mathbf{x}=\mathbf{x}(t ; \overline{\mathbf{x}}, \bar{t})$ be the integral curve of (52) through the point $(\overline{\mathbf{x}}, \bar{t})$. Denote $\mathbf{x}_{0}(\overline{\mathbf{x}}, \bar{t}):=\mathbf{x}(0 ; \overline{\mathbf{x}}, \bar{t})$, then, at $t=0$, the curve passes through the point $\left(\mathbf{x}_{0}(\overline{\mathbf{x}}, \bar{t}), 0\right)$. Recall that, for smooth solutions, the first equation of the system (9) can be reformulated as $\frac{\mathcal{D}(\rho W)}{\mathcal{D} t}=-\rho W \nabla \cdot \mathbf{v}$. Integrating this equation along the curve $\mathbf{x}=\mathbf{x}(t ; \overline{\mathbf{x}}, \bar{t})$ gives

$$
\rho W(\overline{\mathbf{x}}, \bar{t})=\rho_{0} W_{0}\left(\mathbf{x}_{0}(\overline{\mathbf{x}}, \bar{t})\right) \exp \left(-\int_{0}^{\bar{t}} \nabla \cdot \mathbf{v}(\mathbf{x}(t ; \overline{\mathbf{x}}, \bar{t}), t) d t\right)>0,
$$

which, along with $W(\overline{\mathbf{x}}, \bar{t}) \geq 1$, imply $\rho(\overline{\mathbf{x}}, \bar{t})>0$ for all $(\overline{\mathbf{x}}, \bar{t}) \in \mathbb{R}^{3} \times \mathbb{R}_{+}$. For smooth solutions of the modified RMHD system (9), one can derive that $\frac{\mathcal{D}\left(p \rho^{-\Gamma}\right)}{\mathcal{D} t}=0$, which implies $p \rho^{-\Gamma}(\overline{\mathbf{x}}, \bar{t})=$ $p_{0} \rho_{0}^{-\Gamma}\left(\mathbf{x}_{0}(\overline{\mathbf{x}}, \bar{t})\right)>0$. It follows that $p(\overline{\mathbf{x}}, \bar{t})>0$ for any $(\overline{\mathbf{x}}, \bar{t}) \in \mathbb{R}^{3} \times \mathbb{R}_{+}$. It is shown in [36] that, for smooth solutions of (9), the quantity $\frac{\nabla \cdot \mathbf{B}}{\rho W}$ is constant along the curve $\mathbf{x}=\mathbf{x}(t ; \overline{\mathbf{x}}, \bar{t})$, which implies (12). The proof is complete.

\section{REFERENCES}

[1] D. S. BALSARA AND J. KIM, A subluminal relativistic magnetohydrodynamics scheme with ADER-WENO predictor and multidimensional Riemann solver-based corrector, J. Comput. Phys., 312 (2016), pp. 357-384. 
[2] D. S. BAlsara And D. SpICER, A staggered mesh algorithm using high order Godunov fluxes to ensure solenoidal magnetic fields in magnetohydrodynamic simulations, J. Comput. Phys., 149 (1999), pp. 270-292.

[3] A. J. Christlieb, Y. Liu, Q. Tang, And Z. Xu, Positivity-preserving finite difference weighted ENO schemes with constrained transport for ideal magnetohydrodynamic equations, SIAM J. Sci. Comput., 37 (2015), pp. A1825-A1845.

[4] B. Cockburn, S. Hou, And C.-W. Shu, The Runge-Kutta local projection discontinuous Galerkin finite element method for conservation laws. IV. The multidimensional case, Math. Comp., 54 (1990), pp. 545-581.

[5] B. CockBurn And C.-W. Shu, Tvb runge-kutta local projection discontinuous Galerkin finite element method for conservation laws. II. General framework, Math. Comp., 52 (1989), pp. 411-435.

[6] A. Dedner, F. Kemm, D. Kröner, C.-D. Munz, T. Schnitzer, and M. Wesenberg, Hyperbolic divergence cleaning for the MHD equations, J. Comput. Phys., 175 (2002), pp. 645-673.

[7] L. Del Zanna, O. Zanotti, N. Bucciantini, And P. Londrillo, Echo: a Eulerian conservative high-order scheme for general relativistic magnetohydrodynamics and magnetodynamics, Astron. \& Astrophys., 473 (2007), pp. 11-30.

[8] J. Du AND C.-W. Shu, Positivity-preserving high-order schemes for conservation laws on arbitrarily distributed point clouds with a simple WENO limiter, Int. J. Numer. Anal. Model., 15 (2018), pp. 1-25.

[9] C. R. Evans And J. F. Hawley, Simulation of magnetohydrodynamic flows: a constrained transport method, Astrophys. J., 332 (1988), pp. 659-677.

[10] P. Fu, F. LI, AND Y. XU, Globally divergence-free discontinuous Galerkin methods for ideal magnetohydrodynamic equations, J. Sci. Comput., 77 (2018), pp. 1621-1659.

[11] S. K. Godunov, Symmetric form of the equations of magnetohydrodynamics, Numerical Methods for Mechanics of Continuum Medium, 1 (1972), pp. 26-34.

[12] S. Gottlieb, C.-W. Shu, And E. TAdmor, Strong stability-preserving high-order time discretization methods, SIAM Rev., 43 (2001), pp. 89-112.

[13] P. He AND H. TANG, An adaptive moving mesh method for two-dimensional relativistic magnetohydrodynamics, Comput. Fluids, 60 (2012), pp. 1-20.

[14] X. Y. Hu, N. A. Adams, And C.-W. Shu, Positivity-preserving method for high-order conservative schemes solving compressible Euler equations, J. Comput. Phys., 242 (2013), pp. 169-180.

[15] S. S. Komissarov, A Godunov-type scheme for relativistic magnetohydrodynamics, Mon. Not. R. Astron. Soc., 303 (1999), pp. 343-366.

[16] L. Krivodonova, J. Xin, J.-F. Remacle, N. Chevaugeon, and J. E. Flaherty, Shock detection and limiting with discontinuous Galerkin methods for hyperbolic conservation laws, Appl. Numer. Math., 48 (2004), pp. 323-338.

[17] F. Li And C.-W. Shu, Locally divergence-free discontinuous Galerkin methods for MHD equations, J. Sci. Comput., 22 (2005), pp. 413-442.

[18] F. Li, L. Xu, And S. Yakovlev, Central discontinuous Galerkin methods for ideal MHD equations with the exactly divergence-free magnetic field, J. Comput. Phys., 230 (2011), pp. 4828-4847.

[19] C. Liang AND Z. XU, Parametrized maximum principle preserving flux limiters for high order schemes solving multi-dimensional scalar hyperbolic conservation laws, J. Sci. Comput., 58 (2014), pp. 41-60.

[20] D. Ling, J. Duan, And H. TAng, Physical-constraints-preserving Lagrangian finite volume schemes for one-and two-dimensional special relativistic hydrodynamics, J. Comput. Phys., 396 (2019), pp. 507-543.

[21] A. Mignone And G. Bodo, An HLLC riemann solver for relativistic flows-II. magnetohydrodynamics, Mon. Not. R. Astron. Soc., 368 (2006), pp. 1040-1054.

[22] K. G. Powell, An approximate Riemann solver for magnetohydrodynamics (that works in more than one dimension), Tech. Report ICASE Report No. 94-24, NASA Langley, VA, 1994.

[23] K. G. Powell, P. L. Roe, T. J. Linde, T. I. Gombosi, and D. L. D. Zeeuw, A solution-adaptive upwind scheme for ideal magnetohydrodynamics, J. Comput. Phys., 154 (1999), pp. $284-309$

[24] T. Qin, C.-W. Shu, AND Y. YANG, Bound-preserving discontinuous Galerkin methods for relativistic hydrodynamics, J. Comput. Phys., 315 (2016), pp. 323-347.

[25] J. QIU AND C.-W. SHU, Runge-Kutta discontinuous Galerkin method using WENO limiters, SIAM J. Sci. Comput., 26 (2005), pp. 907-929.

[26] D. Radice, L. Rezzolla, And F. Galeazzi, High-order fully general-relativistic hydrodynamics: new approaches and tests, Classical and Quantum Gravity, 31 (2014), p. 075012.

[27] C.-W. SHu, Bound-preserving high-order schemes for hyperbolic equations: Survey and recent developments, in Theory, Numerics and Applications of Hyperbolic Problems II, C. Klingenberg and M. Westdickenberg, eds., Cham, 2018, Springer International Publishing, pp. 591-603.

[28] Z. Sun AND C.-W. Shu, Strong stability of explicit Runge-Kutta time discretizations, SIAM J. Numer. Anal., 57 (2019), pp. 1158-1182.

[29] M. TORRILHON, Locally divergence-preserving upwind finite volume schemes for magnetohydrodynamic equations, SIAM J. Sci. Comput., 26 (2005), pp. 1166-1191.

[30] G. Tо́тн, The $\nabla \cdot \mathbf{B}=0$ constraint in shock-capturing magnetohydrodynamics codes, J. Comput. Phys., 161 (2000), pp. 605-652.

[31] B. van Der Holst, R. Keppens, and Z. Meliani, A multidimensional grid-adaptive relativistic magnetofluid code, Comput. Phys. Commun., 179 (2008), pp. 617-627.

[32] F. Vilar, C.-W. Shu, and P.-H. Maire, Positivity-preserving cell-centered lagrangian schemes for multimaterial compressible flows: From first-order to high-orders. Part II: the two-dimensional case, J. Comput. 
Phys., 312 (2016), pp. 416-442.

[33] K. Wu, Design of provably physical-constraint-preserving methods for general relativistic hydrodynamics, Phys. Rev. D, 95 (2017), 103001.

[34] K. Wu, Positivity-preserving analysis of numerical schemes for ideal magnetohydrodynamics, SIAM J. Numer. Anal., 56 (2018), pp. 2124-2147.

[35] K. Wu AND C.-W. Shu, A provably positive discontinuous Galerkin method for multidimensional ideal magnetohydrodynamics, SIAM J. Sci. Comput., 40 (2018), pp. B1302-B1329.

[36] K. WU AND C.-W. SHU, Entropy symmetrization and high-order accurate entropy stable numerical schemes for relativistic mhd equations, submitted to SIAM J. Sci. Comput., available from arXiv:1907.07467, (2019).

[37] K. Wu AND C.-W. Shu, Provably positive high-order schemes for ideal magnetohydrodynamics: analysis on general meshes, Numer. Math., 142 (2019), pp. 995-1047.

[38] K. WU AND H. TANG, High-order accurate physical-constraints-preserving finite difference WENO schemes for special relativistic hydrodynamics, J. Comput. Phys., 298 (2015), pp. 539-564.

[39] K. Wu AND H. TANG, Admissible states and physical-constraints-preserving schemes for relativistic magnetohydrodynamic equations, Math. Models Methods Appl. Sci., 27 (2017), pp. 1871-1928.

[40] K. Wu AND H. TANG, Physical-constraint-preserving central discontinuous Galerkin methods for special relativistic hydrodynamics with a general equation of state, Astrophys. J. Suppl. Ser., 228 (2017), 3.

[41] K. WU AND H. TANG, On physical-constraints-preserving schemes for special relativistic magnetohydrodynamics with a general equation of state, Z. Angew. Math. Phys., 69 (2018), 84.

[42] Z. Xu, Parametrized maximum principle preserving flux limiters for high order schemes solving hyperbolic conservation laws: one-dimensional scalar problem, Math. Comp., 83 (2014), pp. 2213-2238.

[43] Z. XU AND Y. LiU, New central and central discontinuous galerkin schemes on overlapping cells of unstructured grids for solving ideal magnetohydrodynamic equations with globally divergence-free magnetic field, J. Comput. Phys., 327 (2016), pp. 203-224.

[44] O. ZanotTi, F. FAMBri, AND M. DumbSER, Solving the relativistic magnetohydrodynamics equations with ADER discontinuous Galerkin methods, a posteriori subcell limiting and adaptive mesh refinement, Mon. Not. R. Astron. Soc., 452 (2015), pp. 3010-3029.

[45] X. Zhang, On positivity-preserving high order discontinuous Galerkin schemes for compressible Navier-Stokes equations, J. Comput. Phys., 328 (2017), pp. 301-343.

[46] X. Zhang And C.-W. Shu, On maximum-principle-satisfying high order schemes for scalar conservation laws, J. Comput. Phys., 229 (2010), pp. 3091-3120.

[47] X. Zhang And C.-W. Shu, On positivity-preserving high order discontinuous Galerkin schemes for compressible Euler equations on rectangular meshes, J. Comput. Phys., 229 (2010), pp. 8918-8934.

[48] X. Zhang, Y. XIA, AND C.-W. Shu, Maximum-principle-satisfying and positivity-preserving high order discontinuous galerkin schemes for conservation laws on triangular meshes, J. Sci. Comput., 50 (2012), pp. 29-62.

[49] J. Zhao And H. TANG, Runge-Kutta discontinuous Galerkin methods for the special relativistic magnetohydrodynamics, J. Comput. Phys., 343 (2017), pp. 33-72.

[50] S. Zou, X. YU, AND Z. DAI, A positivity-preserving Lagrangian discontinuous Galerkin method for ideal magnetohydrodynamics equations in one-dimension, J. Comput. Phys., 405 (2020), p. 109144. 